\title{
SUSTAINED INTRA-CARTILAGE DELIVERY OF LOW DOSE DEXAMETHASONE USING A CATIONIC CARRIER FOR TREATMENT OF POST TRAUMATIC OSTEOARTHRITIS
}

A.G. Bajpayee ${ }^{1,2}$, R.E. De la Vega ${ }^{3,4}$, M. Scheu ${ }^{3,5}$, N.H. Varady ${ }^{2}$, I.A. Yannatos ${ }^{2}$, L.A. Brown ${ }^{3}$, Y. Krishnan ${ }^{2}$, T.J. Fitzsimons ${ }^{3}$, P. Bhattacharya ${ }^{2}$, E.H. Frank ${ }^{2}$, A.J. Grodzinsky ${ }^{2,6, \S}$ and R.M. Porter ${ }^{3,7, *, \S}$

${ }^{1}$ Departments of Bioengineering and Mechanical Engineering, Northeastern University, 360 Huntington Avenue, Boston, MA 02115, USA

${ }^{2}$ Centre for Biomedical Engineering, MIT, 77 Massachusetts Avenue, Cambridge, MA 02139, USA

${ }^{3}$ Department of Orthopaedic Surgery, Beth Israel Deaconess Medical Centre (BIDMC),

Harvard Medical School, 330 Brookline Avenue, Boston, MA 02215, USA

${ }^{4}$ Current affiliation: Rehabilitation Medicine Research Centre, Mayo Clinic, 200 First St. SW, Rochester, MN 55905, USA

${ }^{5}$ Department of Orthopaedic Surgery, Clínica Alemana de Santiago, Avenida Vitacura 5951, Vitacura, Chile

${ }^{6}$ Departments of Biological, Mechanical and Electrical Engineering, MIT, 77 Massachusetts Avenue, Cambridge, MA 02139, USA

${ }^{7}$ Current affiliation: Departments of Internal Medicine and Orthopaedic Surgery, University of Arkansas for Medical Sciences, 4301 W. Markham Street, Little Rock, AR 72205, USA $\S$ These authors contributed equally

\begin{abstract}
Disease-modifying osteoarthritis drugs (DMOADs) should reach their intra-tissue target sites at optimal doses for clinical efficacy. The dense, negatively charged matrix of cartilage poses a major hindrance to the transport of potential therapeutics. In this work, electrostatic interactions were utilised to overcome this challenge and enable higher uptake, full-thickness penetration and enhanced retention of dexamethasone (Dex) inside rabbit cartilage. This was accomplished by using the positively charged glycoprotein avidin as nanocarrier, conjugated to Dex by releasable linkers. Therapeutic effects of a single intra-articular injection of low dose avidin-Dex (0.5 mg Dex) were evaluated in rabbits 3 weeks after anterior cruciate ligament transection (ACLT). Immunostaining confirmed that avidin penetrated the full cartilage thickness and was retained for at least 3 weeks. Avidin-Dex suppressed injury-induced joint swelling and catabolic gene expression to a greater extent than free Dex. It also significantly improved the histological score of cell infiltration and morphogenesis within the periarticular synovium. Micro-computed tomography confirmed the reduced incidence and volume of osteophytes following avidin-Dex treatment. However, neither treatment restored the loss of cartilage stiffness following ACLT, suggesting the need for a combinational therapy with a pro-anabolic factor for enhancing matrix biosynthesis. The avidin dose used caused significant glycosaminoglycan (GAG) loss, suggesting the use of higher Dex : avidin ratios in future formulations, such that the delivered avidin dose could be much less than that shown to affect GAGs. This charge-based delivery system converted cartilage into a drug depot that could also be employed for delivery to nearby synovium, menisci and ligaments, enabling clinical translation of a variety of DMOADs.
\end{abstract}

Keywords: Intra-cartilage drug delivery, cartilage, electrostatics, dexamethasone, post-traumatic osteoarthritis, rabbit anterior cruciate ligament transection, avidin.

*Address for correspondence: Ryan M. Porter, University of Arkansas for Medical Sciences, Division of Endocrinology and Metabolism, 4301 W. Markham Street, Mail Slot \#587, Little Rock, AR 72205 Telephone : +1 5015266990 Email:rmporter@uams.edu

\section{Introduction}

Post-traumatic osteoarthritis (PTOA) accounts for $12 \%$ of the total osteoarthritis (OA) cases (Brown et al., 2006). Following a traumatic joint injury, the levels of inflammatory cytokines (e.g. IL-1, IL-6, TNF $\alpha$ ) significantly increase in the synovial fluid (Kapoor et al., 2011; Swärd et al., 2012). This can predispose even undamaged cartilage to rapid proteolysis and loss of aggrecan and other matrix molecules, within the first days to weeks post-injury (Anderson et al., 2011). Often accompanied by damage to other 
joint tissues, such injuries lead to PTOA with high incidence. Potential disease-modifying OA drugs (DMOADs), including anti-catabolic glucocorticoids (Grodzinsky et al., 2017; Huebner et al., 2014) and proanabolic growth factors (Fortier et al., 2001; Miller et al., 2010), have been identified as being able to reverse or prevent PTOA. However, to date, clinical trials evaluating DMOAD candidates have failed due to systemic toxicity or lack of evidence of sustained benefit and/or effective cartilage targeting (Evans et al., 2014).

There is a critical need for new drug delivery systems, which can increase joint residence times and enhance target tissue specificity, thereby increasing treatment effectiveness and reducing systemic toxicity. Following joint injury, direct intra-articular (IA) injection is indicated to treat localised pain and inflammation. However, drugs are cleared rapidly from the joint (Evans et al., 2014; Larsen et al., 2008), so that patients must either receive multiple injections or settle for what short term benefit can be achieved with a single injection. Drug delivery to targets inside articular cartilage is further challenged by the dense extracellular matrix (ECM) of this tissue that is mainly composed of collagen and aggrecan, which is a proteoglycan containing highly negatively charged glycosaminoglycan (GAG) chains. The tightly-packed organisation of GAGs hinders the ability of drugs to penetrate and diffuse within the cartilage (Bajpayee et al., 2014b). It is critical for drugs to penetrate the full depth of cartilage in order to reach the chondrocytes and ECM targets involved in OA pathogenesis (Evans, 2016). Drug carrier systems proposed so far, including polymeric microparticles (Bodick et al., 2015), liposomes (Dong et al., 2013) and drug-loaded hydrogels (Kimmerling et al., 2015; Petit et al., 2015), have prolonged joint residence time due to their large size or aggregating properties; however, they are not able to penetrate into and through the joint cartilage. While these drug carriers can be useful in providing extended pain and inflammation relief through enhanced delivery to targets in the synovium, preclinical and clinical studies strongly suggest that certain DMOADs must be delivered to chondrocytes or ECM components to achieve cartilage protection (Chevalier et al., 2009; Cohen et al., 2011). As a first step toward addressing this challenge, avidin, due to its optimal size and positive charge (Bajpayee et al., 2014b), can penetrate the full thickness of rabbit cartilage following intra-articular administration, resulting in an intra-cartilage half-life of about $7 \mathrm{~d}$ (Bajpayee et al., 2015). Such cationic proteins and their derivatives offer great potential as carriers for intracartilage drug delivery. Moreover, by creating a drug depot within the cartilage, this approach may be used to target other joint tissues known to be involved in the progression of PTOA (Bajpayee and Grodzinsky, 2017).

Dexamethasone (Dex), a broad-spectrum corticosteroid, has potent anti-inflammatory effects, enhances chondrocyte differentiation and proteoglycan synthesis and reduces glycosaminoglycan loss in injured cartilage in vitro (Grodzinsky et al., 2017; Li et al., 2015; Lu et al., 2011) and in vivo (Huebner et al., 2014; Malfait et al., 2009). However, the precise actions of corticosteroids are highly dependent on the cumulative dose applied, complicating their therapeutic assessment. Huebner et al. (2014) demonstrate that frequent, intra-articular injection of Dex $(0.5 \mathrm{mg} / \mathrm{kg}$ or about $2.5 \mathrm{mg}$ administered every $3 \mathrm{~d}$ ) in a bone-drill rabbit model of PTOA significantly reduce inflammation and protected cartilage; however, this high multidose regimen causes systemic side effects in many vital organs, demonstrating the critical need for targeted, low dose therapy. In recent years, clinical trials have been initiated to evaluate the ability of sustained release formulations of glucocorticoids (e.g. triamcinolone), encapsulated within polymeric particles (NCT02357459; Flexion Therapeutics, Burlington, VT, USA) or conjugated with hyaluronic acid (NCT02022930; Carbylan Therapeutics, Palo Alto, CA, USA), to suppress joint pain and inflammation, but such systems naturally use high drug doses (40$60 \mathrm{mg}$ of drug).

In the present study, the disease-modifying effects of a relatively low dose of Dex $(0.5 \mathrm{mg})$ was evaluated, delivered by a single injection using avidin (Av) as nanocarrier, in a rabbit anterior cruciate ligament transection (ACLT) model of PTOA. Dex was conjugated with avidin using a combination of the following two linkers to provide sustained drug release over several days: ester, a fast release linker, and hydrazone, an acid-cleavable, slow release linker. A single initial low dose of Av-Dex conjugate effectively suppresses GAG loss from cultured cartilage explants treated continuously with IL-1 over a 3-week period (Bajpayee et al., 2016). In this single dose condition, cartilage explants are subjected to Dex or Av-Dex for only the first $2 \mathrm{~d}$ and subsequent medium changes do not contain Dex, thereby simulating a single intra-articular injection of Dex in vivo. Using this approach, elicits a sustained biological response similar to continuous administration of free (unconjugated) Dex, in which the culture medium is replenished with Dex every $2 \mathrm{~d}$. In addition, a single dose of Av-Dex has a significantly greater and prolonged effect in suppressing cytokineinduced aggrecanolysis (GAG loss) over the 3 weeks treatment period compared to a single dose of free Dex (Bajpayee et al., 2016).

The main goal of this study was to assess the therapeutic effect of a single, low dose of Av-Dex, injected IA 3 weeks after ACLT in rabbits, and compare it with saline, free (unconjugated) Dex and avidin alone. The working hypothesis was that intra-cartilage delivery of low dose Dex using avidin nanocarriers could suppress injury-induced catabolic activity in the rabbit ACLT model of PTOA after just one IA injection into the knee. The severity of arthritic changes in cartilage and bone of rabbit knee joints was assessed by quantitative reverse 
transcription polymerase chain reaction (qRT-PCR), immunohistochemistry (IHC) and biochemical assays to measure GAG and collagen concentration in articular cartilage and by dynamic mechanical analyses to evaluate changes in tissue biomechanical properties. Micro-computed tomography $(\mu \mathrm{CT})$ was used to assess changes in peri-articular bone properties, including osteophyte formation.

\section{Materials and Methods}

\section{Formulation of Av-Dex}

$4 \mathrm{~mol}$ of dexamethasone [molecular weight (MW) 390 Da] were conjugated with 1 mol of avidin (MW 66 kDa) by using a 1:1 molar ratio of ester and $\mathrm{pH}$ sensitive hydrazone linkers (all reagents were purchased from Sigma Aldrich, St. Luis, MO, USA), as described previously (Bajpayee et al., 2016). This formulation of $\mathrm{Av}$-Dex was selected as it provides the longest sustained release of Dex over a period of 3 weeks (Bajpayee et al., 2016). Av-Dex was synthesised by a two-step reaction protocol. In the first step, Dex was conjugated to biotinylated poly(ethyleneglycol) (PEG, $2.3 \mathrm{kDa}$ ) through either an ester or a hydrazone linker. In the second step, biotinylated PEG-Dex conjugate was supramolecularly attached to avidin by using its four biotin binding sites. The drug loading content (DLC) in the Av-Dex conjugate was estimated to be about $2 \%$ by weight measurement and HPLC (Agilent 1200 series, Agilent Technologies Inc, CA, USA), as described previously (Bajpayee et al., 2016). Since the current design of conjugate incorporated $4 \mathrm{~mol}$ of Dex onto $1 \mathrm{~mol}$ of avidin, a theoretical drug loading content of $2.3 \%$ for fully conjugated avidin was expected. As a result, $20 \mathrm{mg}$ of avidin were required to deliver $0.5 \mathrm{mg}$ Dex to rabbit knee joints. Av-Dex was constituted in sterile distilled water and frozen at $-20^{\circ} \mathrm{C}$ until further use.

\section{Animals}

Animal studies were performed as pre-approved by the Institutional Animal Care and Use Committee at Beth Israel Deaconess Medical Centre (Boston, MA, USA).

The residence time for avidin (and other solutes) in cartilage is proportional to the square of cartilage thickness (Bajpayee et al., 2015). Since cartilage thickness is related to joint size, the use of animals larger than mice or rats is important for generating intra-cartilage drug residence times that more closely approximate what would occur in humans. Thus, for a PTOA model, 12 months old, mature, female New Zealand rabbits with an average weight of $5 \mathrm{~kg}$, purchased from Millbrook Breeding Labs (Amherst, MA, USA), were used. Due to the unanticipated closure of this vendor during the study, the final ten rabbits were ordered from an alternate vendor, Covance Research Products (Denver, PA, USA). The thickness of rabbit tibial cartilage was measured to be between 350-550 $\mu \mathrm{m}$ (Bajpayee et al., 2015), consistent with previously reported data (Hoch et al., 1983). Generally, by 8 months of age, the growth plates in the distal femur and proximal tibia are closed, thereby defining skeletal maturity (Hunziker et al., 2007; Masoud et al., 1986). The closed physes inhibit regeneration of rabbit articular cartilage once degradation sets in (Gregory et al., 2012), which is necessary for proper evaluation of biological efficacy and mechanisms of therapeutic intervention.

\begin{tabular}{|l|l|l|l|}
\hline $\boldsymbol{n}=\mathbf{1 0}$ each group & \multicolumn{1}{|c|}{ Group name } & $\begin{array}{c}\text { Left contralateral } \\
\text { knee }\end{array}$ & \multicolumn{1}{|c|}{ Right ACLT knee } \\
\hline Group 1 & Saline 3 weeks & Saline (non-operated) & Saline \\
\hline Group 2 & Dex 3 weeks & Saline (sham) & Dex \\
\hline Group 3 & Av-Dex 3 weeks & Saline (sham) & Av-Dex \\
\hline Group 4 & Av 3 weeks & Avidin (sham) & Avidin \\
\hline Group 5 & Saline 9 weeks & Saline (sham) & Saline \\
\hline
\end{tabular}

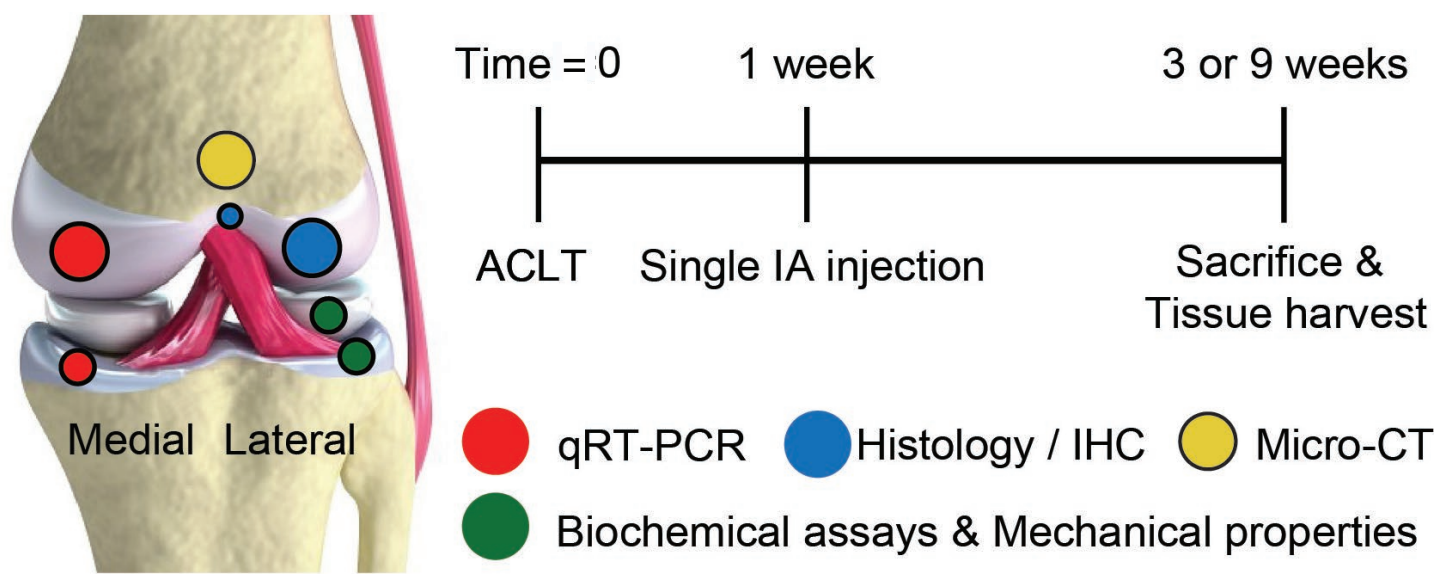

Fig. 1. The five treatment groups, timelines of study procedures and distribution of joint tissues across a variety of assays are shown. 


\section{In vivo study design}

Five groups of 10 rabbits each (Fig. 1) were used to study the biological efficacy of a single dose, sustained-release formulation of Av-Dex compared to injection of a single dose of soluble unconjugated Dex, in an ACLT model of PTOA. Unilateral ACLT was performed in the right knees of these 50 rabbits. Under isoflurane anaesthesia, the rabbit hind limbs were shaved and scrubbed with betadine and ethanol. The joint space was accessed through a medial parapatellar incision, the patella was dislocated laterally and the knee flexed. The ACL was secured with a Frazier micro-dissecting Dura hook (Harvard Apparatus, Holliston, MA, USA) and transected at the approximate mid-length using a \#15 scalpel blade, taking care not to injure the surrounding structures. After flushing the joint space with sterile saline solution, the knee was extended, the patella was repositioned and an anterior drawer test was performed to confirm complete ACL transection. Except for group 1, the contralateral (left) knees underwent sham surgery, consisting of opening the joint capsule, visually confirming the intact ACL and flushing the joint space with saline solution. Group 1 contralateral left knees were used as non-operated controls. Following ACLT or sham procedure, the joint capsule was closed with interrupted sutures and the skin was closed with subcutaneous running sutures to prevent the rabbits from chewing-open the incision site. Upon recovery, rabbits were allowed free cage activity until the experimental endpoint.

At day 7 post-surgery, the rabbits were again anaesthetised and each knee received an IA injection of $600 \mu \mathrm{L}$, as detailed in Fig. 1. Group 1 (saline 3 weeks) received saline solution in both knees. Group
2 (Dex 3 weeks) received soluble Dex $(0.5 \mathrm{mg})$ in the right knees and saline solution in the left knees. Group 3 (Av-Dex 3 weeks) received Av-Dex (total Dex dose of $0.5 \mathrm{mg}$ ) in the right knees and saline solution in the left knees. Group 4 (Av 3 weeks) received avidin (20 mg; equivalent dose required to deliver $0.5 \mathrm{mg}$ of Dex) in both knees to evaluate its effect on both the contralateral sham knee as well as the ACLT knee. Groups 1 to 4 were euthanised at week 3. Group 5 (saline 9 weeks) received saline solution in both knees and was euthanised at week 9 - when more substantive osteoarthritic changes are reported in the rabbit ACLT model (Batiste et al., 2004; Bouchgua et al., 2009b; Chang et al., 1997; Hashimoto et al., 2002; Yoshioka et al., 1996) - in order to serve as a reference group for longer term changes. After IA injection using an anterolateral approach, both knees were slowly flexed several times to distribute the injected solution uniformly throughout the joint space.

\section{Selection of Dex dose}

A single dose of Av-Dex, with an effective Dex concentration of $100 \mu \mathrm{M}$, significantly suppresses cytokine-induced GAG loss over 3 weeks in vitro (Bajpayee et al., 2016). Based on the fact that there is about $1 \mathrm{~mL}$ of synovial fluid in the inflamed rabbit joint and $\sim 35-40 \%$ of IA injected avidin is retained inside the joint after $24 \mathrm{~h}$ (Bajpayee et al., 2015), a total of $0.15 \mathrm{mg}$ of Dex is needed to achieve a $100 \mu \mathrm{M}$ concentration inside the rabbit joint $24 \mathrm{~h}$ after IA injection. The glucocorticoid dose recommended for small animals, such as cats and dogs, is $0.5 \mathrm{mg}$ per $5 \mathrm{~kg}$ weight. Thus, a low, one-time dose of $0.5 \mathrm{mg}$ Dex was used.

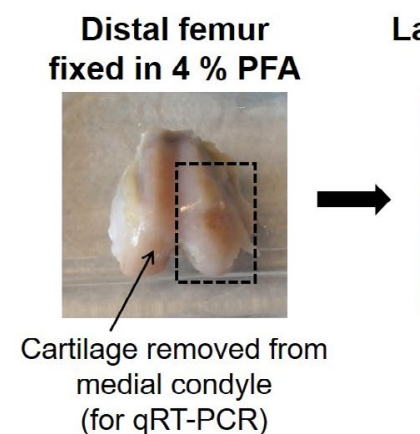

Lateral femoral condyle (post-EDTA decal)

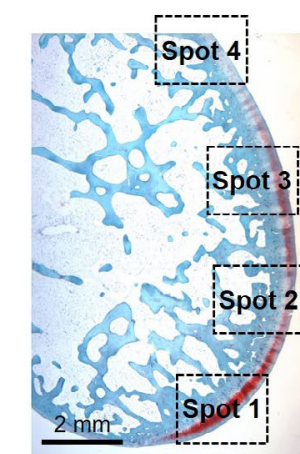

Stitched image of

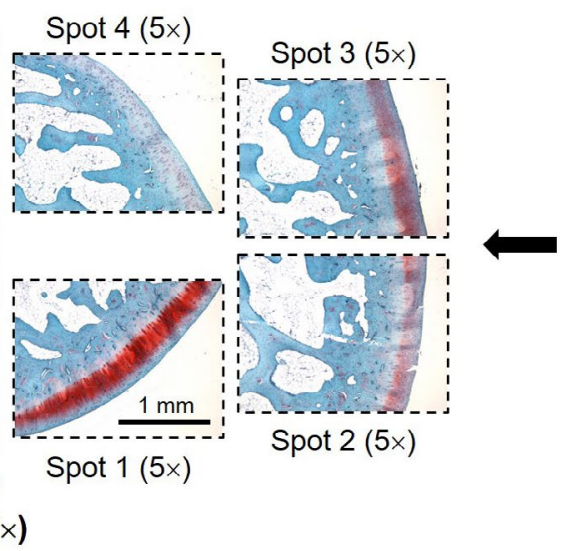

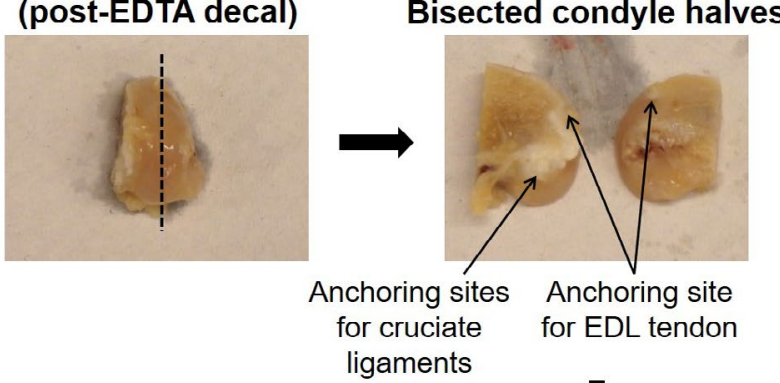

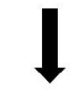

Paraffin block

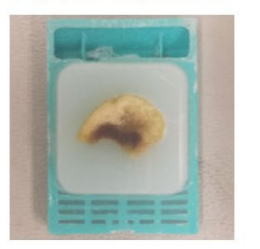

Fig. 2. The lateral femoral condyles of rabbit distal femora were decalcified in EDTA, bisected and the halves embedded in paraffin. Sagittal sections of the condyle halves were stained with safranin $\mathrm{O} /$ fast green and haematoxylin. Stitched images of the whole surface, as well as four highermagnification images, were scored according to the system of Laverty et al. (2010): the stitched images were used to score GAG staining and surface structure, while the higher magnification images were used to determine chondrocyte density and clustering. 


\section{Joint effusion and osteophyte formation}

Using a digital caliper, the medial-lateral joint width was measured at the mid-length of the patellar tendon before surgery (day 0 ), on the day of IA injection (1 week) and at the time of euthanasia ( 3 or 9 weeks), as an approximate measure of joint effusion and osteophyte formation (Fig. 2). Percentage change in knee width at 1 or $3 / 9$ weeks was calculated as follows:

$$
\% C H=\frac{W A-W B}{W A} \times 100 \%
$$

where: $\% \mathrm{CH}=\%$ change in knee width at 1,3 or 9 weeks; WA = Knee width at 1,3 or 9 weeks; $\mathrm{WB}=$ Knee width before surgery. Synovitis and osteophyte formation were confirmed by macroscopic examination, $\mu \mathrm{CT}$ imaging and histological analysis of lateral femoral condyles, as described below.

\section{Tissue harvest and allocation}

After euthanasia, both knee joints were opened and gross morphology was assessed qualitatively for osteophytes, soft tissue fibrillation and lesions. Full-thickness articular cartilage samples from the medial tibial plateau and medial femoral condyle were collected using a sterile scalpel blade and pooled as 'medial cartilage'. The medial articular cartilage and the medial menisci were "flash" frozen in liquid nitrogen for gene expression analysis by qRTPCR. Next, the distal femora, with intact cartilage on the lateral femoral condyles, were harvested and fixed for 1 week in $4 \%$ paraformaldehyde in phosphate-buffered saline (PBS) for $\mu \mathrm{CT}$ followed by histology and immunohistochemistry. The lateral tibial plateau cartilage (with intact tibial bone) and the lateral menisci were frozen in PBS containing proteinase inhibitors (cOmplete ${ }^{\mathrm{TM}}$ Protease Inhibitor Cocktail tablet in $50 \mathrm{~mL}$ PBS, Roche Applied Science, Indianapolis, IN, USA) for measurement of mechanical properties followed by biochemical assays to measure GAG and collagen concentration in these tissues. Table 1 describes the distribution of joint tissues for the outcome measures detailed below. $\mu \mathrm{CT}$

After fixation, the distal femora were scanned using a desktop $\mu \mathrm{CT}$ imaging system ( $\mu \mathrm{CT} 40$; Scanco Medical AG, Brüttisellen, Switzerland). Scans were acquired using a $70 \mathrm{kV}$ x-ray tube potential, $114 \mu \mathrm{A}$ intensity, $300 \mathrm{~ms}$ integration time, $30 \mu \mathrm{m}$ isotropic voxel size and a phantom calibration of $700 \mathrm{mg}$ hydroxyapatite (HA)/ $\mathrm{cm}^{3}$. Analysis was performed between the epiphyseal line proximally and the condyles distally. A volume of interest was defined, which encompassed only trabecular bone using a predetermined algorithm (Bouxsein et al., 2010). The following measurements were obtained: volumetric trabecular bone mineral density [BMD; mg HA/ $\mathrm{cm}^{3}$ ], trabecular bone volume fraction (bone volume/ total volume; BV/TV), trabecular number (Tb.N), mean thickness (Tb.Th; mm), mean separation (Tb. sp; mm), structural model index (SMI), quantifying the trabecular bone structure as either more rod- or plate-like (SMI values range from 0 for a perfect plate to 3 for a perfect rod, where negative values indicate concave trabecular surfaces) (Florea et al., 2015), and degree of anisotropy (DA), as an indicator of how the trabecular bone structural elements are oriented (Bouxsein et al., 2010).

Osteophyte volume was determined by manually outlining ectopic bone in each consecutive $\mu \mathrm{CT}$ slice on the base of its reduced bone mineral density and deviation from the margins of normal bone. This was used to create 3D models of osteophytic bone for quantitative volumetric assessment. Osteophyte severity from $3 \mathrm{D} \mu \mathrm{CT}$ models of the tibia for each knee was graded on a 4 point scale by three investigators (RDLV, TJF and AGB) in a blinded fashion, where $0=$ no observed osteophyte, $1=$ local and less severe osteophytes, 2 = widespread, but moderately severe osteophytes and 3 = widespread and very severe osteophytes (Jones et al., 2010; Shirai et al., 2011).

\section{Gene expression using qRT-PCR}

The articular cartilage from the medial compartment was used for PCR analysis, as the medial side of the joint is reported to be more sensitive to early changes

Table 1. Distribution of joint tissues across assays.

\begin{tabular}{|c|c|c|c|c|c|c|c|}
\hline \multirow[b]{2}{*}{ Assays } & \multicolumn{2}{|c|}{ Medial side } & \multicolumn{3}{|c|}{ Lateral side } & \multirow[b]{2}{*}{$\begin{array}{c}\begin{array}{c}\text { Patella } \\
\text { cartilage }\end{array} \\
\end{array}$} & \multirow[b]{2}{*}{$\begin{array}{c}\text { Trochlear } \\
\text { groove }\end{array}$} \\
\hline & $\begin{array}{l}\text { Femoral } \\
\text { condyle }\end{array}$ & $\begin{array}{c}\text { Tibia } \\
\text { plateau }\end{array}$ & $\begin{array}{l}\text { Femoral } \\
\text { condyle }\end{array}$ & $\begin{array}{c}\text { Tibia } \\
\text { plateau }\end{array}$ & Meniscus & & \\
\hline qRT-PCR & $x$ & $X$ & & & & & \\
\hline$\mu \mathrm{CT}$ & $x$ & & $x$ & & & & $x$ \\
\hline Histology/IHC & & & $x$ & & & & \\
\hline $\begin{array}{l}\text { Mechanical } \\
\text { properties }\end{array}$ & & & & $X$ & $x$ & & \\
\hline $\begin{array}{l}\text { GAG and } \\
\text { collagen }\end{array}$ & & & & $X$ & $x$ & $X$ & \\
\hline
\end{tabular}


Table 2. A scoring system was developed to assess synovial infiltration and morphogenesis at the perienthesis region shown in Fig. 4a-j. The composite scores are presented in Fig. $4 \mathbf{k}$.

\begin{tabular}{|c|l|}
\hline \multicolumn{2}{|l|}{ Peri-enthesis score } \\
\hline \multicolumn{2}{|c|}{ I. Synovial infiltration (0-3): } \\
\hline 0 & No infiltration with normal cartilage \\
\hline 1 & Little synoviocyte infiltration and/or chondrocyte proliferation \\
\hline 2 & Mild synoviocyte infiltration (replacing partial thickness of cartilage) \\
\hline 3 & Severe synoviocyte infiltration (replacing full thickness of cartilage) \\
\hline \multicolumn{2}{|c|}{ II. Morphogenesis (0-3): } \\
\hline 0 & No chondrogenesis by synovial cells; subchondral remodelling of articular surface \\
\hline 1 & Isolated synovial chondrometaplasia; osseous encroachment beyond tidemark \\
\hline 2 & Extensive synovial chondrometaplasia, with or without isolated ossification \\
\hline 3 & Extensive ossification of synovial infiltrate with new haematopoiesis \\
\hline
\end{tabular}

in PTOA models (Le Graverand et al., 2002). These samples were pulverised, while still frozen, using a TissueLyser II homogeniser (Qiagen, Germantown, IL, USA). Total RNA was extracted with an RNeasy Mini kit (Qiagen) using the TRI Reagent (Molecular Research Centre, Cincinnati, OH, USA) and cDNA was generated using a High-Capacity cDNA Reverse Transcription Kit (Applied Biosystems, Foster City, CA, USA). The following transcripts were measured by qRT-PCR using pre-validated rabbit $\mathrm{RT}^{2}$ qPCR Primer Assays and $\mathrm{RT}^{2}$ SYBR Green qPCR Mastermix (Qiagen): cartilage matrix components type II collagen alpha1 chain (COL2A1; \#PPN00317A), aggrecan core protein (ACAN; PPN00382A), pro-inflammatory cytokines interleukin$1 \beta$ (IL1B; PPN00304A), tumour necrosis factor- $\alpha$ (TNFA; PPN00391A), matrix metalloproteinases-1 (MMP1; PPN00411A), matrix metalloproteinases-3 (MMP3; PPN00412A), matrix metalloproteinases-13 (MMP13; PPN00075A) and a disintegrin and metalloproteinase with thrombospondin motifs 5 (ADAMTS5; PPN00159A). Gene expression was normalised using glyceraldehyde-3-phosphate dehydrogenase (GAPDH; PPN00377A) and compared to contralateral controls using the $2^{-\triangle \Delta C T}$ method (Livak and Schmittgen, 2001). Very low GAPDH levels indicated insufficient mRNA yield/quality from the majority of avidin-alone samples, so this group was excluded from analysis.

\section{Tissue biomechanical properties and concentration of GAGs and collagen}

The lateral tibial plateau cartilage (with intact tibial bone) and the lateral menisci were thawed at room temperature and their biomechanical properties were measured using a Dynastat mechanical spectrometer (IMASS, Hingham, MA, USA), adapting the method described by Hoch et al. (1983). A 1 mm diameter non-porous plane-ended indenter, fixed to the Dynastat's load cell assembly, was used to test three spots on the lateral tibial plateau and one spot on the lateral meniscus, respectively. At each spot on the tibial plateau surface, four consecutive displacements were applied to achieve steps-in strain of $5 \%, 5 \%, 2.5 \%$ and $2.5 \%$, for a total of $15 \%$ strain. For the lateral meniscus, four consecutive displacements of $10 \%$ strain each were applied for a total strain of $40 \%$. After each step-in strain, the equilibrium stress (measured load normalised to indenter area) was recorded after stress relaxation reached equilibrium and the equilibrium stiffness was calculated as the best fit to the linear regression of the resultant equilibrium stress-strain curve. At $15 \%$ (tibial cartilage) or $40 \%$ (meniscus) final static strain, sinusoidal displacements corresponding to $0.5 \%$ dynamic strain amplitude were superposed on the static offset strain at frequencies ranging from $0.005 \mathrm{~Hz}$ to $2 \mathrm{~Hz}$. Thereby, the dynamic stiffness was calculated from the ratio of dynamic stress and strain amplitudes, as a function of frequency. The meniscus samples were subjected to higher compressive strains than the cartilage to probe their deep layers, where most of proteoglycans were concentrated. Thereafter, these cartilage and menisci samples were digested using Proteinase K (Roche, Indianapolis, IN, USA) and total GAG and collagen content were measured using the dimethylmethylene blue (DMMB) assay and the hydroxyproline assay, respectively (Li et al., 2015). GAG and collagen content data are expressed as $\mu \mathrm{g}$ per mg of tissue wet weight.

\section{Histology and immunohistochemistry}

After being scanned for $\mu \mathrm{CT}$ analysis, the distal femora were bisected longitudinally and the lateral condyles were decalcified for 8 weeks using $15 \%$ EDTA in PBS ( $\mathrm{pH} 7.4$ ), changing decalcification solution twice weekly. After the first week, the lateral condyles were bisected in a sagittal plane to improve penetration of the decalcification solution. $6 \mu \mathrm{m}$ thick sagittal sections from each condyle half were rehydrated and stained using $0.5 \%$ safranin $\mathrm{O}, 0.01 \%$ fast green and Weigert's iron haematoxylin (Schmitz et al., 2010). The image were acquired using a Leica DMLB microscope (Leica Microsystems, Buffalo Grove, IL, USA). equipped with a digital camera (Qimaging, Surrey, British Columbia, Canada), stitching individual 1.6× magnification images (using Image-Pro; Media Cybernetics, Rockville, MD, USA) to produce a composite image of the articular surface. These stitched images and four higher magnification 
(5x) images, taken from specific locations along the condylar surface (Fig. 2), were scored according to the system recommended by the OARSI histopathology initiative (Laverty et al., 2010). This system accounts for GAG staining and surface integrity along the articular surface as well as chondrocyte density and clustering. Both GAG staining and surface integrity were evaluated in the stitched (1.6x) images, while cell density and clustering were scored in the four $5 \times$ magnification images. Scoring was performed by three different investigators (AGB, LAB and and RMP) in a blinded fashion and then averaged; interreviewer variation was $<20 \%$ for all categories. For each lateral condyle, mean reviewers scores from the two bisected halves were averaged and the scores for each treatment/control group were presented using box-and-whisker plots, reporting median values with the $25^{\text {th }}-75^{\text {th }}$ quartile range.

During histological scoring, it was observed that synovial infiltration and osteophyte formation could often be visualised near the insertion site (i.e. enthesis) of the extensor digitorum longus (EDL) tendon. $\mathrm{MCT}$ analysis confirmed that osteophytes extended into this region of the condyles. Because the lateral condyles were bisected near the EDL enthesis, this feature was present in most sections and could be used as a reference point for histological evaluation. A custom score was applied to these sections characterising both (i) synovial infiltration and (ii) morphogenesis (i.e. chondrometaplasia and endochondral bone formation) (Table 2). These scores were also presented as median values with $25^{\text {th }}-75^{\text {th }}$ quartile range in box-and-whisker plots.

Following antigen retrieval with $0.1 \%$ hyaluronidase and $0.1 \%$ pronase $(30 \mathrm{~min}$ at $\left.37^{\circ} \mathrm{C}\right)$, sections were immunostained for type II collagen using $1 \mu \mathrm{g} / \mathrm{mL}$ mouse IgG1 (clone II-II6B3; Developmental Studies Hybridoma Bank, University of Iowa, Iowa City, IA, USA) and a VectaStain Elite ABC kit (Vector Laboratories, Burlingame, CA, USA) for detection using 3,3-diaminobenzidine (DAB). To detect residual avidin, additional hyaluronidasedigested sections were stained with $5 \mu \mathrm{g} / \mathrm{mL}$ rabbit anti-avidin IgG directly conjugated to horseradish peroxidase (PA1-26844, Thermo Fisher Scientific, Waltham, MA, USA). This procedure bypassed the need for using biotinylated peroxidase from the VectaStain kits, which could produce higher signal for avidin molecules whose biotin binding sites were not occupied (Fig. 11b,e,h). Staining specificity was determined by substituting the test antibodies with normal mouse or rabbit IgGs. All DAB-stained sections were counterstained with Gill number 2 haematoxylin.

\section{Statistical Analysis}

All data are presented as mean $\pm 95 \%$ confidence intervals (CI), unless specified otherwise. Nonparametric Mann-Whitney U test was performed to compare contralateral control knee and the ACLT knee within each treatment group at 3 and 9 weeks. $p<0.05$ was considered statistically significant. For the synovitis/morphogenesis sub-scores and OARSI histological scores, a Mann-Whitney U test was performed to determine statistical differences between the contralateral control knee and ACLT knee within a group at 3 and 9 weeks. A KruskalWallis ANOVA followed by post hoc Dunn's test was used to compare individual groups against each other at each time point.

\section{Contralateral Control ACLT}
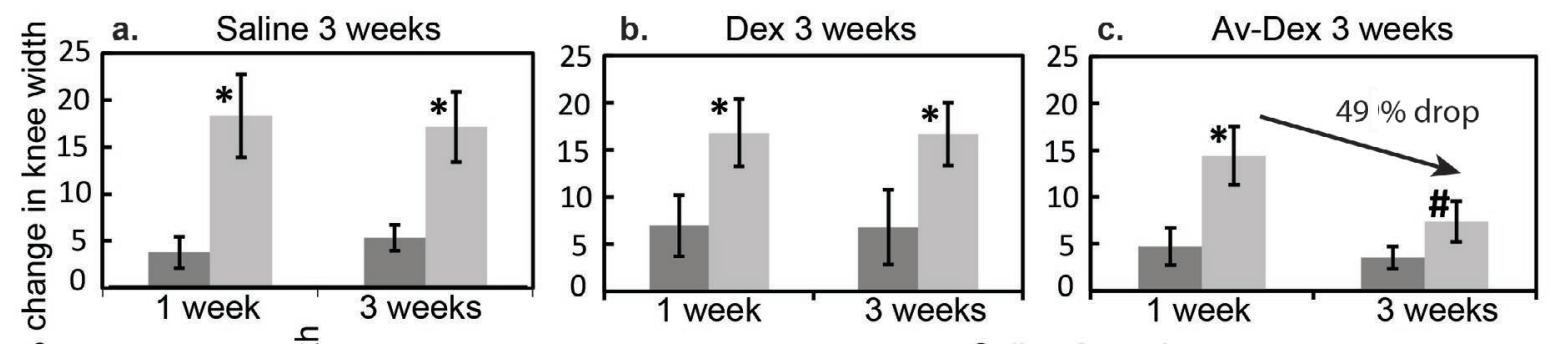

$$
\text { ㅇํำ }
$$
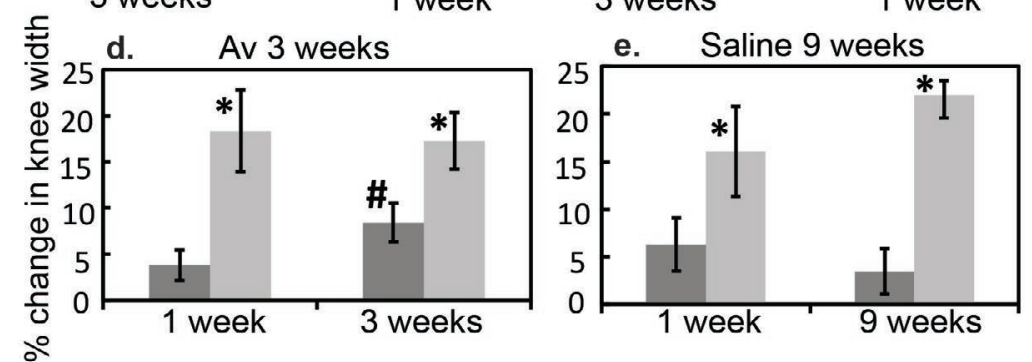

Fig. 3. Percentage change in medial-lateral joint width measured at week 1, i.e. on the day of IA injection, and 3 weeks post-surgery, i.e. at the time of euthanasia (vs. joint width before surgery), as an approximate measure of joint effusion and osteophyte formation. ${ }^{*}$ shows significant difference $(p<0.05)$ between ACLT and contralateral knee within each treatment group; \# shows significant difference $(p=0.01)$ between corresponding values at two different time points. Data are shown as mean $\pm 95 \%$ CI. 


\section{Results}

\section{Joint inflammation and synovitis}

The change in medial-lateral joint width was measured at week 1 (on the day of IA injection) and 3 weeks post-surgery (at the time of euthanasia), as an approximation of joint inflammation and effusion (Fig. 3). In all treatment groups, the width of ACLT joints significantly increased compared to contralateral control knees $(p<0.05)$, 1 week post ACLT; treatment with saline or Dex alone did not relieve joint inflammation at week 3 (Fig. 3a,b). The percentage change in knee width increased from an average value of $17 \%$ at week 3 (Fig. 3a) to $21 \%$ at week 9 in saline-treated groups (Fig. 3e). Av-Dex treatment significantly reduced joint inflammation by $\sim 49 \%$ in the ACLT knee at week $3(p=0.01$, Fig. 3c). Avidin alone did not affect the knee width in the ACLT knee; however, an increase from $3.8 \%$ to $8.4 \%$ at week 3 (Fig. $3 \mathrm{~d}$ ) was observed in the contralateral sham surgical control knees, which also received avidin IA injection. In general, synovial fluid aspirated from the joint space of ACLT knees appeared more viscous and, in some cases, reddish in colour compared to contralateral controls. Macroscopically, the cartilage in ACLT knees seemed generally healthy at 3 weeks; however, by 9 weeks, fissures and full-depth erosions were evident. At 3 weeks, some irregularities in menisci were observed and, by 9 weeks, prominent lesions, more severe in the medial menisci, were present, consistent with previous findings (Boulocher et al., 2007; Chang et al., 1997).

Synovitis and osteophyte formation was observed within ACLT knees at week 3 and worsened by week 9 in the saline group, as also observed by others (Batiste et al., 2004; Bouchgua et al., 2009b). These features were especially prominent along the femoral condyles (Fig. $4 \mathbf{b}, \mathbf{d}$ ), often extending to where the EDL tendon inserted into the lateral condyle. Accordingly, the EDL enthesis was used as a landmark for consistently assessing synovitis and morphogenesis by histology. As shown in Fig. 4a-j, while hyaline cartilage was typically observed proximal to the EDL enthesis in most contralateral knees, significant synoviocyte infiltration (Syn) with varying degrees of chondrometaplasia $(\mathrm{Ch})$ and endochondral ossification (Oss) were observed in ACLT knees injected with saline. This was quantitatively calculated using a custom composite score (Table 2, Fig. 4k). Treatment with Dex alone (ACLT, group 2) did not improve the composite score. In contrast, Av-Dex (ACLT, group 3) resulted in an improved composite score (Fig. 4k), primarily through a reduction in the morphogenesis subscore (Fig. 41). While some synovitis was present in the Av-Dex group, ossification was not observed in most of these specimens (e.g. Fig. 4a-j). Treatment with avidin alone (group 4) did not affect the score for the contralateral control knees (sham, group 4), but resulted in a higher composite score in the ACLT knees (ACLT, group 4) compared to the saline 3 weeks group (ACLT, group 1$)$.

\section{$\mu \mathrm{CT}$ analysis of femoral condyles \\ Osteophyte formation}

Most femoral condyles in the ACLT groups showed osteophyte formation by week 3, except for the AvDex-treated group (Fig. 5c), where only one out of nine joints (Fig. 5f) showed discernible osteophytes with low volume and a low severity score $(0.1 \pm 0.2)$, consistent with the observations of Fig. 4 . The severity of osteophytes worsened by week 9 in the saline group, as reflected in both osteophyte volume (Fig. 5 g) and severity scores (Fig. 5h), in agreement with previous findings (Batiste et al., 2004; Bouchgua et al., 2009b; Bouchgua et al., 2009a). Treatment with Dex or avidin alone did not improve osteophyte volume or severity scores. Avidin treated contralateral left knees showed some osteophyte formation (Fig. 5d), consistent with the observed percentage increase in knee width (Fig. 3).

\section{Trabecular bone}

Table $3 \mathbf{a}, \mathbf{b}$ compared trabecular bone parameters between the contralateral control and ACLT knees of each group. The trabecular bone in the ACLT knees showed statistically lower bone volume fraction (BV/TV 25.3\%, $p=0.02$ ) and trends toward reduced bone mineral density, trabecular number, thickness and increased trabecular spacing compared to contralateral control knees at 3 weeks (Tables 3a,b). These trends became statistically significant by week 9. Similar trends are reported in earlier studies (Bouchgua et al., 2009b; Florea et al., 2015; Pinney et al., 2012; Wang et al., 2007). Treatment with Dex did not restore bone volume fraction, but with Av-Dex, the difference in BV/TV between ACLT and control groups was no longer statistically significant (Table $3 b)$. In the avidin-treated group, the trends observed were similar to the saline 3 weeks group. BMD correlated strongly with BV/TV in all groups. Despite these changes in trabecular structure, no changes were observed in SMI or degree of anisotropy (DI).

\section{qRT-PCR analysis of medial cartilage}

Transcript levels in cartilages from the medial tibial plateau and medial distal femur were measured by qRT-PCR and compared to contralateral controls (Fig. 6). Table 4 summarised statistical comparisons in expression levels between the ACLT knees and contralateral control knees for each group. No statistically significant changes in COL2A1 transcript levels were observed in ACLT knees compared to their respective contralateral controls for any treatment condition. For $A C A N$, decreased transcript expression in the ACLT knee, found for the saline treated groups at 3 weeks and 9 weeks $(p<0.05)$, was consistent with the literature (Hotta et al., 2005; Majima et al., 2002). Treatment with Dex and Av- 


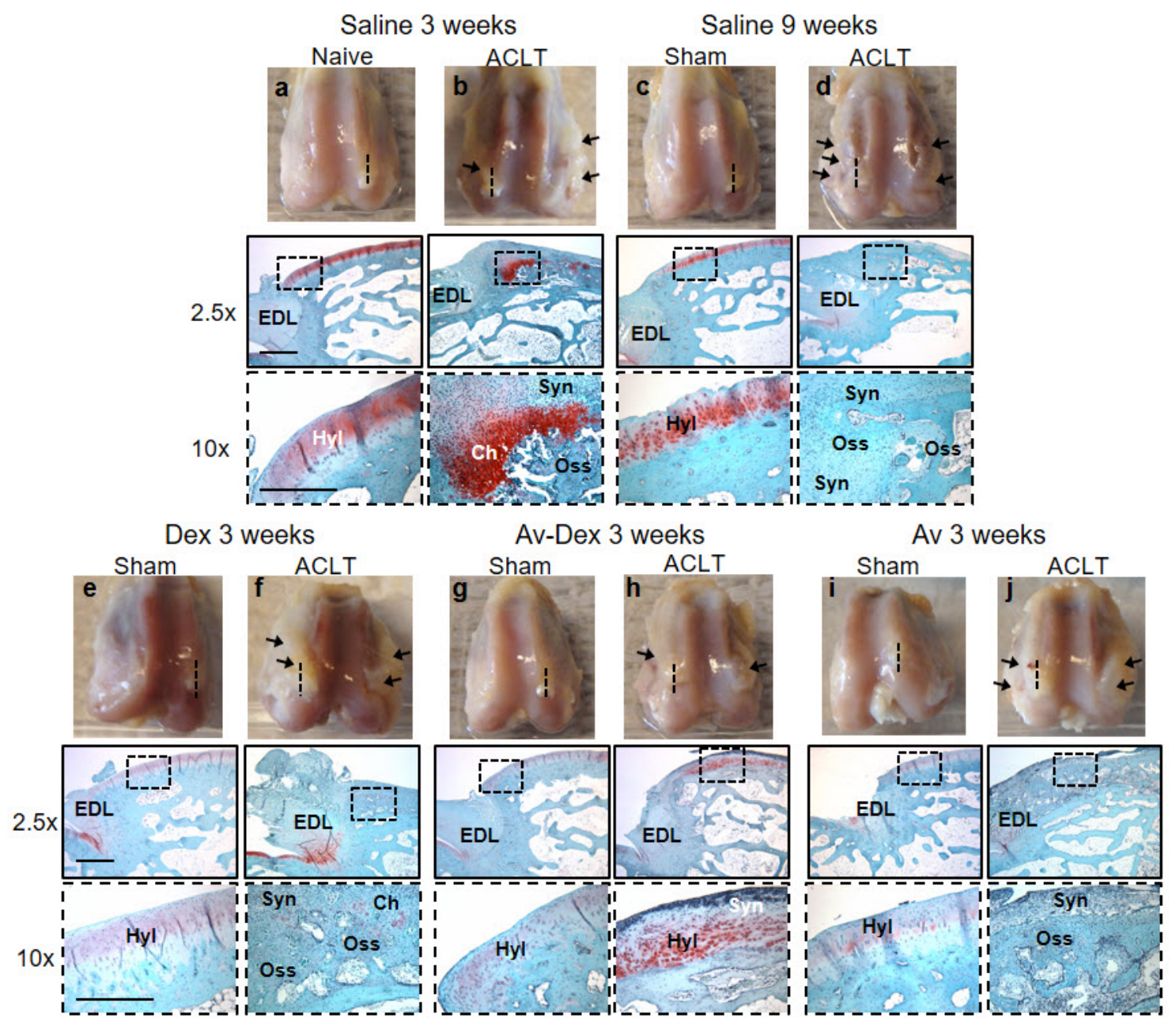

\section{Contralateral control $\quad$ ACLT}

k. Composite score
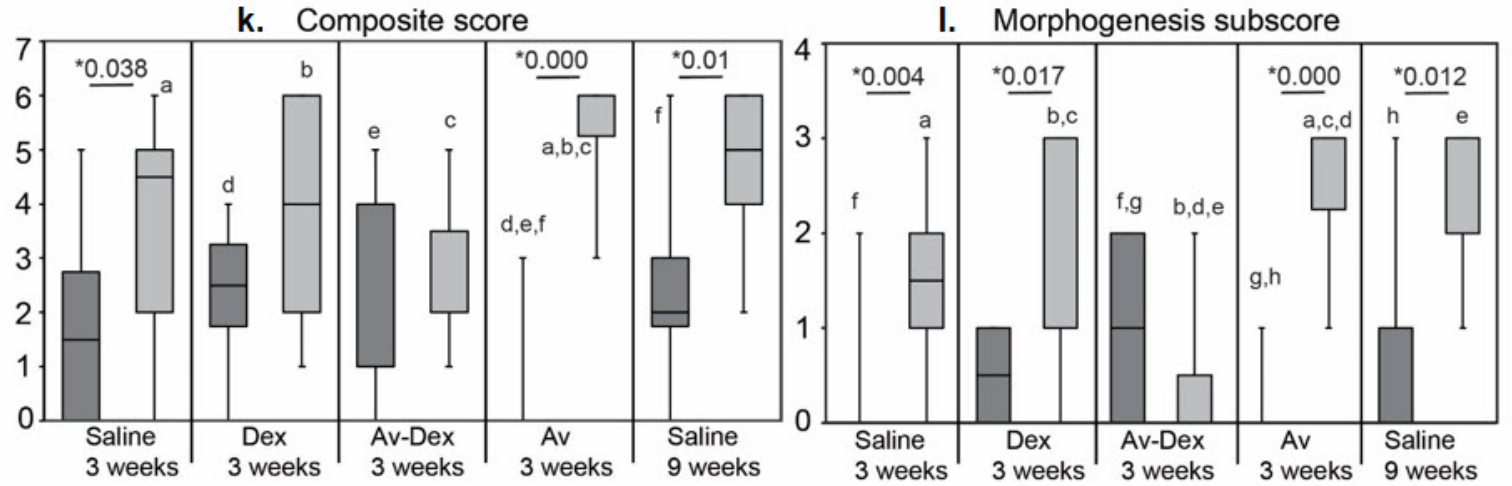

Fig. 4. (a-j) Representative distal femora harvested 3 or 9 weeks after sham surgery or ACLT. Substantial bilateral synovitis and/or osteophyte formation (arrows) could be observed within ACLT knees. The lateral condyles were used for histology, bisecting them in a sagittal plane that cut through the enthesis (insertion site) of the extensor digitorum longus (EDL) tendon. The dotted lines indicate the approximate plane represented in the matched micrographs showing safranin O/fast green staining of the sagittal sections. Lower magnification images (scale bar $=1 \mathrm{~mm}$ ) were acquired near the EDL enthesis, while higher magnification images (insets; scale bar $=200 \mu \mathrm{m}$ ) show the articular surface proximal to the enthesis. While hyaline cartilage (Hyl) could still be observed along the articular surface of control knees, in many ACLT knees, synoviocyte infiltration (Syn), chondrometaplasia (Ch) and endochondral ossification (Oss) were observed in this region. (k) Composite scores assessing synovial infiltration and morphogenesis at the peri-enthesis region shown in panels a-j are reported as box-and-whisker plots for the contralateral control (dark grey) and ACLT (light grey) knees of each treatment group ( $n=7-10)$. Morphogenesis subscore is also shown. Median values with $25^{\text {th }}-75^{\text {th }}$ quartile range are reported. * shows significant difference between ACLT and control within each treatment group using Mann Whitney U test. Groups that share letters within each graph are significantly different from one another $(p<0.05$, Kruskal-Wallis ANOVA followed by post hoc Dunn's test). 
Dex restored $A C A N$ expression to levels that were not significantly different from contralateral control knees.

For the pro-inflammatory cytokine $I L-1 \beta$, transcript levels increased for the saline-treated group at 3 weeks and this trend also held for the group treated with Dex alone $(p<0.05)$. However, treatment with Av-Dex decreased $I L-1 \beta$ expression to levels that matched sham controls. Also expression of MMP1 increased for the saline-treated and Dex- treated groups at 3 weeks $(p<0.05)$. Similar to $I L-1 \beta$, the Av-Dex treatment group showed no statistically significant change in MMP1 levels compared to contralateral knees. For $M M P 3$ and $M M P 13$, transcript levels increased as expected for the saline-treated and Dex-treated groups at 3 weeks and 9 weeks. However, for these genes, increased expression was also observed with Av-Dex treatment $(p<0.05)$. Finally, ADAMTS5 transcript levels were elevated for the Dex-treated group, but in contrast, treatment with
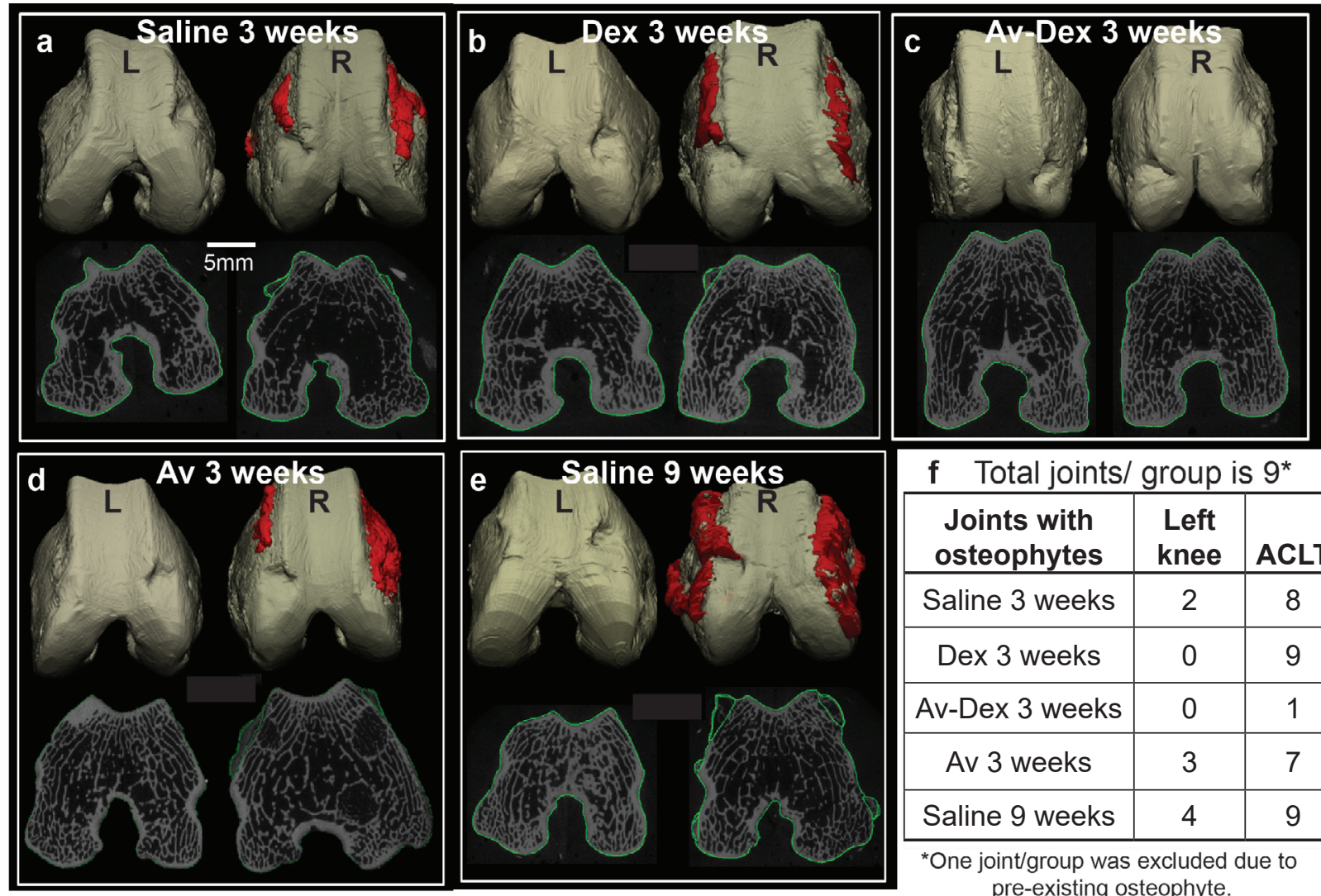

\begin{tabular}{|c|c|c|}
\hline \multicolumn{1}{c|}{ Total joints/ group is ${ }^{*}$} \\
\hline $\begin{array}{c}\text { Joints with } \\
\text { osteophytes }\end{array}$ & $\begin{array}{c}\text { Left } \\
\text { knee }\end{array}$ & ACLT \\
\hline Saline 3 weeks & 2 & 8 \\
\hline Dex 3 weeks & 0 & 9 \\
\hline Av-Dex 3 weeks & 0 & 1 \\
\hline Av 3 weeks & 3 & 7 \\
\hline Saline 9 weeks & 4 & 9 \\
\hline
\end{tabular}

*One joint/group was excluded due to

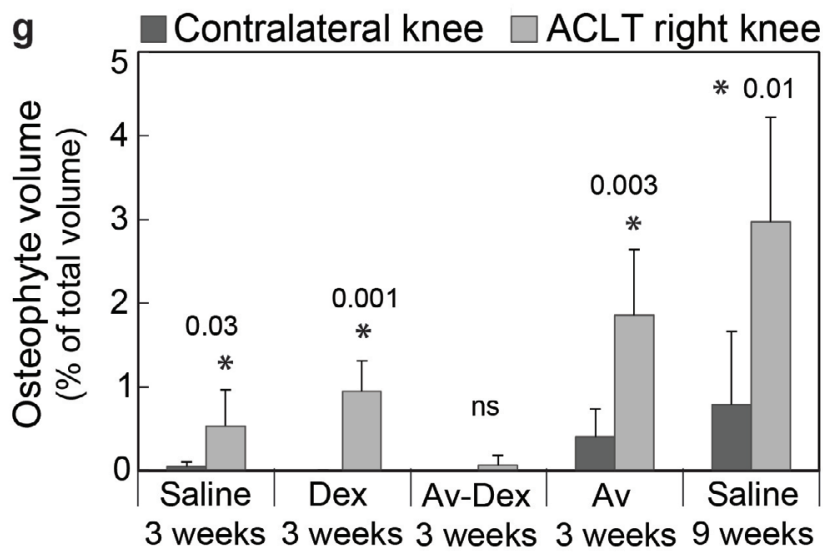

h
\begin{tabular}{|c|c|c|}
\hline $\begin{array}{c}\text { Osteophyte } \\
\text { severity }\end{array}$ & $\begin{array}{c}\text { Left knee } \\
\text { Mean } \pm 95 \% \text { CI }\end{array}$ & ACLT \\
\hline $\begin{array}{c}\text { Saline } \\
3 \text { weeks }\end{array}$ & $0.2 \pm 0.2$ & $1.1 \pm 0.3$ \\
\hline Dex 3 weeks & $0.0 \pm 0.0$ & $1.6 \pm 0.3$ \\
\hline $\begin{array}{c}\text { Av-Dex } \\
\text { 3 weeks }\end{array}$ & $0.0 \pm 0.0$ & $0.1 \pm 0.2$ \\
\hline $\begin{array}{c}\text { Av } \\
\text { weeks }\end{array}$ & $0.4 \pm 0.4$ & $1.6 \pm 0.6$ \\
\hline $\begin{array}{c}\text { Saline } \\
9 \text { weeks }\end{array}$ & $0.6 \pm 0.6$ & $2.8 \pm 0.3$ \\
\hline
\end{tabular}

Fig. 5. 3D reconstructions of rabbit femoral condyles to show osteophytic bone growth for quantitative volumetric assessment in the following treatment groups: (a) saline 3 weeks, (b) Dex 3 weeks, (c) AvDex 3 weeks, (d) Av 3 weeks and (e) saline 9 weeks. Condyles on the left side of each image were from contralateral left knees (L) and those on the right side were from the experimental ACLT knees (R). Representative images are shown. (f) Number of joints out of nine in each treatment group that showed osteophyte formation. (g) Osteophyte volume expressed as percentage of total volume. Data are shown as mean $\pm 95 \%$ CI. * shows significant difference $(p<0.05)$ between ACLT and contralateral knee within each treatment group. NS = not significant. (h) Osteophyte severity score where $0=$ no observed osteophyte, $1=$ local and less severe osteophytes, 2 = widespread but moderately severe osteophytes and $3=$ widespread and very severe osteophytes. 


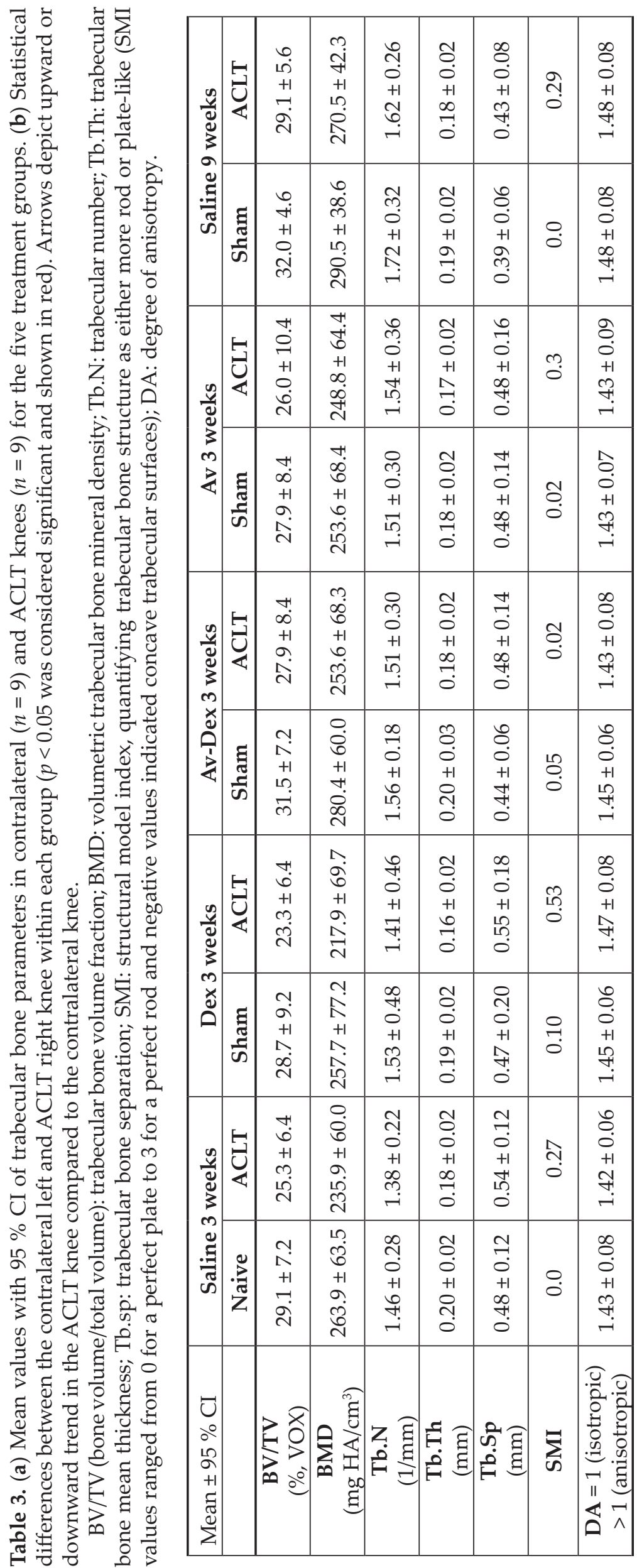

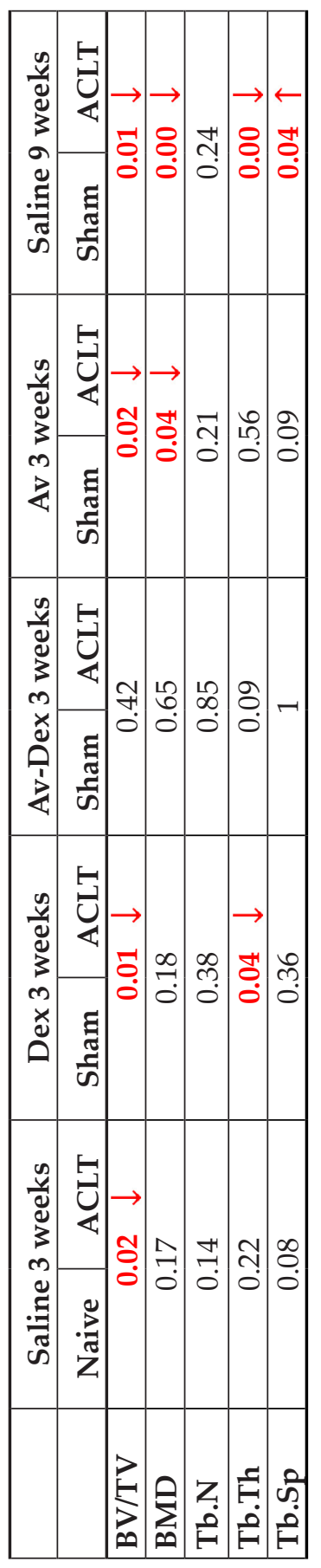


Av-Dex decreased ADAMTS5 expression relative to contralateral controls $(p<0.05)$. In all groups, Av-Dex was able to restore normal gene expression levels for ACAN, IL-1 $\beta, M M P 1$ and ADAMTS5.

\section{Mechanical and compositional analyses of lateral tibial cartilage and meniscus}

The effective equilibrium indentation stiffness of lateral tibial cartilage was significantly reduced by almost a factor of two in the ACLT knees compared to the contralateral knees (Fig. 7b). Treatment with Dex or Av-Dex did not restore this equilibrium stiffness by week 3 . Avidin alone did not affect the cartilage stiffness in the contralateral sham knees compared to the contralateral 3 weeks saline group. At week 9, the equilibrium stiffness in the cartilage from ACLT knees was greater compared to contralateral control knees. Similar trends were observed in dynamic stiffness (Fig. 7c,d). No significant differences were observed in collagen content of the tibial cartilage from ACLT
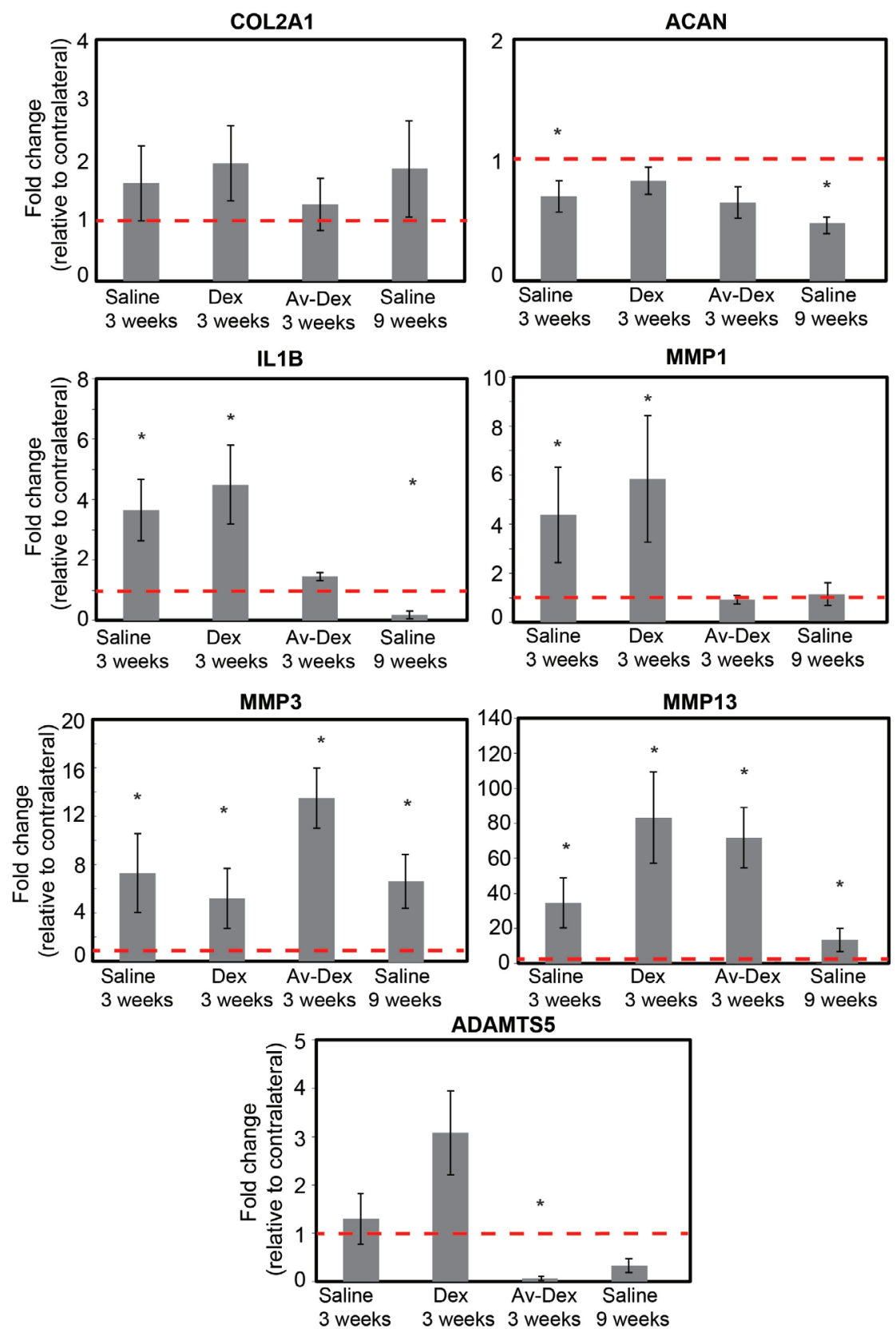

knees and contralateral knees for all treatment conditions (Fig. 7e). A significant reduction in GAG concentration was observed in the tibial cartilage of ACLT knees compared to the contralateral knees for all groups except saline 9 weeks (Fig. 7f). For patellar cartilage, a significant increase in collagen content was observed only for saline 9 weeks $(p=0.0004$, Fig. 7g); in contrast, a significant decrease in GAG concentration was measured in the ACLT knee cartilage compared to contralateral controls for all treatment groups except saline 9 weeks (Fig. 7h). For the lateral meniscus, a significant decrease in both equilibrium and dynamic stiffness was measured at 9 weeks (Fig. 8b-d); no differences between ACLT menisci and contralateral controls for any of the 3-week conditions was observed. In addition, there were no differences observed in collagen or GAG concentration in the menisci for any of the treatment groups (Fig. 8e,f).
Fig. 6. Relative mRNA levels from pooled medial tibial and femoral cartilage in the ACLT knee normalised to contralateral knee for each treatment condition. Data shown as mean $\pm 95 \%$ CI. * shows significant difference $(p<0.05$, exact $p$-values shown in Table 4) compared to contralateral knee within each treatment group. $n=9-10$ joints in each group. 
Table 4. Summary of changes in mRNA levels in the experimental ACLT knee compared to the contralateral knee for each treatment group. $p$-values represent statistical differences between the contralateral left and ACLT right knee within each group. Significant values $(p<0.05)$ are shown in red. Arrows depict upward or downward trend in the ACLT knee compared to the contralateral knee.

\begin{tabular}{|c|c|c|c|c|c|c|c|c|}
\hline \multirow{2}{*}{$\begin{array}{l}\text { Pooled cartilage from } \\
\text { medial side } \\
\text { (tibial + femoral) }\end{array}$} & \multicolumn{2}{|c|}{ Saline 3 weeks } & \multicolumn{2}{|c|}{ Dex 3 weeks } & \multicolumn{2}{|c|}{ Av-Dex 3 weeks } & \multicolumn{2}{|c|}{ Saline 9 weeks } \\
\hline & Naive & ACLT & Sham & ACLT & Sham & ACLT & Sham & ACLT \\
\hline COL2A1 & \multicolumn{2}{|c|}{0.19} & \multicolumn{2}{|c|}{0.06} & \multicolumn{2}{|c|}{0.64} & \multicolumn{2}{|c|}{0.19} \\
\hline$A C A N$ & \multicolumn{2}{|c|}{$0.01 \downarrow$} & \multicolumn{2}{|c|}{0.94} & \multicolumn{2}{|c|}{0.20} & \multicolumn{2}{|c|}{0.00} \\
\hline$I L-1 \beta$ & \multicolumn{2}{|c|}{$0.01 \uparrow$} & \multicolumn{2}{|c|}{$0.00 \uparrow$} & \multicolumn{2}{|c|}{0.14} & \multicolumn{2}{|c|}{0.03} \\
\hline MMP1 & \multicolumn{2}{|c|}{$0.02 \uparrow$} & \multicolumn{2}{|c|}{$0.00 \uparrow$} & \multicolumn{2}{|c|}{0.51} & \multicolumn{2}{|c|}{0.41} \\
\hline MMP3 & \multicolumn{2}{|c|}{$0.00 \uparrow$} & \multicolumn{2}{|c|}{$0.00 \uparrow$} & \multicolumn{2}{|c|}{$0.00 \uparrow$} & \multicolumn{2}{|c|}{$0.01 \uparrow$} \\
\hline MMP13 & \multicolumn{2}{|c|}{$0.01 \uparrow$} & \multirow{2}{*}{\multicolumn{2}{|c|}{$\begin{array}{ll}0.00 & \uparrow\end{array}$}} & \multirow{2}{*}{\multicolumn{2}{|c|}{$\begin{array}{ll}0.00 & \uparrow \\
& \end{array}$}} & \multicolumn{2}{|c|}{$0.01 \uparrow$} \\
\hline ADAMTS5 & \multicolumn{2}{|c|}{0.72} & & & 0.01 & & \multicolumn{2}{|c|}{0.24} \\
\hline
\end{tabular}

\section{Histology and immunohistochemistry of lateral femoral condyles}

Sagittal sections from the lateral femoral condyles were stained with safranin $\mathrm{O} /$ fast green and used together with an OARSI-recommended scoring system (Laverty et al., 2010) to grade GAG staining, surface structure along the condyle, chondrocyte density and clustering. Variation in these properties along the length of the condyles contributed to the overall variance reported in the four sub-scores (Fig. 9a-d). Stainings from representative specimens (i.e. at or near the median composite score) are shown in Fig. 10. Some loss of safranin O staining in the superficial cartilage was observed for not only ACLT, but also contralateral knees, which may be attributed to preexisting degenerative changes in these 12 months old rabbits.

At week 3, ACLT knees were characterised by regional areas of partial to full thickness GAG loss and structural defects, particularly superficial fissuring, compared to contralateral controls. These changes were generally more extensive by week 9 , particularly in the middle third of the condyle surface (Fig. 10g insert). However, when the total condyle surface was considered, safranin $\mathrm{O}$ and structure sub-scores were not significantly different from 3 to 9 weeks (Fig. 9a,b). Rather, the greatest change between 3 and 9 weeks was in chondrocyte clustering, which increased for saline-treated ACLT knees (Fig. 9d). Treatment with Dex alone did not significantly influence any of the sub-scores compared to salinetreated knees. Surface structure was not significantly influenced by Av-Dex or avidin alone compared to 3 weeks saline (Fig. 9b). Trends toward decreased chondrocyte density (i.e. increased sub-scores) were measured for Av-Dex and avidin alone (Fig. 9c); however, chondrocyte clustering was not affected. Unexpectedly, Av-Dex treatment led to marked loss of safranin O staining throughout the full thickness of most condyles from this group (Fig. 10o), translating into significantly higher safranin $\mathrm{O}$ sub-scores (Fig. 9a). Of note, avidin alone also produced full thickness reductions in safranin $\mathrm{O}$ staining for not only ACLT knees, but also most contralateral knees, which were as well injected with avidin (Fig. 9a; 10q,s). This could suggest that the dose of avidin used in this study $(20 \mathrm{mg}$ ) caused GAG loss within the condylar cartilage. Alternatively, if significant avidin levels remained within the articular cartilage, this could inhibit binding of the cationic safranin $\mathrm{O}$ dye to GAGs. Immunostaining for type II collagen suggests that any changes in GAG levels did not correspond to loss of this key collagen component by week 3 (Fig. $10)$.

To detect residual avidin within the articular surface 3 weeks post-surgery ( 2 weeks post-injection), additional sections were immunostained using a peroxidase-conjugated antibody specific for avidin. Sensitivity of this detection protocol was confirmed using bovine cartilage disks pre-incubated for $24 \mathrm{~h}$ in 10 or $100 \mu \mathrm{M}$ avidin (Fig. 11a,d,g). Specificity was confirmed by staining rabbit lateral condyles from knees not treated with avidin: these specimens, including the contralateral knees from the AvDex treated group, did not produce significant DAB staining (Fig. 11j,k). In contrast, staining was detected within the articular cartilage of ACLT knee treated with Av-Dex (Fig. 11n,o) or avidin alone (Fig. $11 \mathbf{v}, \mathbf{w})$, as well as those contralateral knees injected with avidin (Fig. 11r,s). While the intensity of immunostaining generally correlated with the intensity of safranin $\mathrm{O}$ staining in adjacent sections, some immunostaining was also seen in regions of low safranin O staining, leaving open the possibility of reduced safranin $\mathrm{O}$ sensitivity by residual avidin. However, incubating healthy bovine cartilage disks for $24 \mathrm{~h}$ with $100 \mu \mathrm{M}$ avidin did not reduce subsequent safranin $\mathrm{O}$ staining intensity (Fig. 11j). Avidin immunostaining was also detected at the EDL enthesis, within the GAG-rich tendon anchoring site (not shown). While the articular surface proximal to the EDL enthesis often contained extensive chondrometaplasia (as in Fig. 4a), immunostaining was primarily observed beneath these regions of new chondrogenesis, near the location of the original articular surface (Fig. 11o,w; arrows). 


\section{Discussion}

The working hypothesis was that intra-cartilage delivery of low dose Dex using avidin as nanocarrier could suppress injury-induced catabolic activity in a rabbit ACLT model of PTOA, using just one injection. It was found that a single injection of AvDex significantly suppressed ACLT-induced joint inflammation by $49 \%$ at week 3 (Fig. 3c), despite the low dose of Dex $(0.5 \mathrm{mg})$ used. Free Dex, on the other hand, did not relieve joint inflammation (Fig. 3b), most likely owing to its short joint residence time. Av-Dex significantly improved the composite score evaluating synoviocyte infiltration and morphogenesis along the lateral femoral condyle (Fig. 4 b) by inhibiting chondrometaplasia and formation
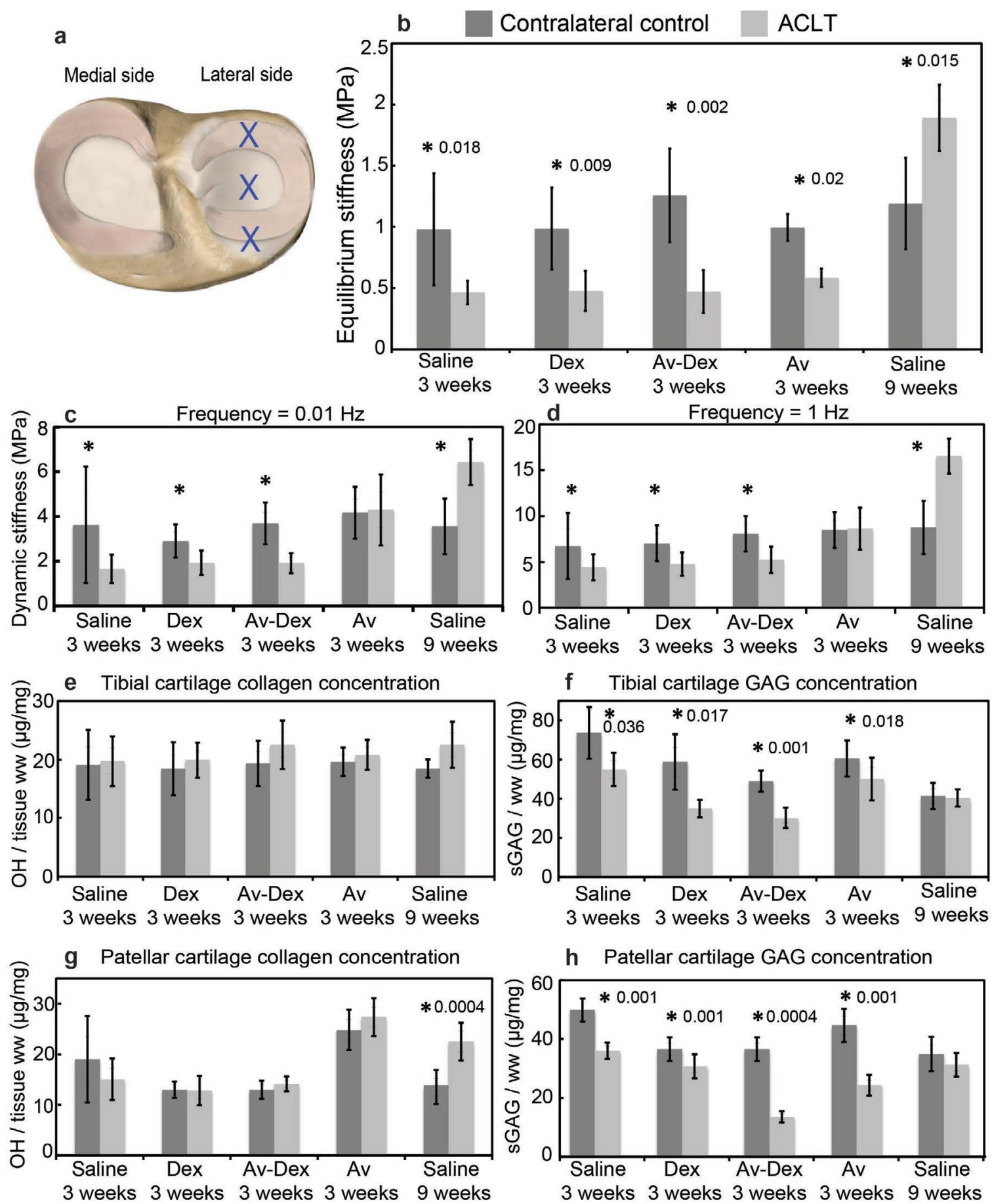

Fig. 7. Mechanical and biochemical properties of lateral tibial plateau articular cartilage. (a) Blue crosses represent the locations tested on the lateral tibial plateau cartilage. (b) Average equilibrium stiffness values of lateral tibial cartilage from both contralateral control and ACLT knees for each treatment group. Average dynamic stiffness measured in tibial cartilage at (c) $0.01 \mathrm{~Hz}$ and (d) $1 \mathrm{~Hz}$. (e) Collagen concentration measured using the hydroxyproline assay and (f) GAG concentration measured using DMMB assay in lateral tibial cartilage. (g) Collagen concentration and (h) GAG concentration in patellar cartilage. Data are shown as mean $\pm 95 \% \mathrm{CI}$; ${ }^{*}$ represents significant difference $(p<0.05)$ between ACLT and contralateral knee within each treatment group. $n=9-10$ joints in each group. 
of osteophytes. This inhibition of endochondral ossification by Av-Dex translated into reduced incidence and volume of osteophytes, as shown by $\mu \mathrm{CT}$ (Fig. 5c,f,h). Free Dex, on the other hand, did not induce any improvement, again likely due to its rapid clearance from the joint. Most of the animals in the Dex group showed osteophytes with severity similar to the saline 3 weeks group (Fig. 5b). Factors like IL-1, TNF- $\alpha$, TGF $\beta$ are believed to be involved in inflammation-mediated osteogenesis within the joint (Jones et al., 2010; van der Kraan and van den Berg, 2007) and they can be suppressed by sustained doses of glucocorticoids (Huebner et al., 2014; Kumar et al., 2014; Sieker et al., 2016; Wernecke et al., 2015). Additionally, $\mu \mathrm{CT}$ assessment of trabecular bone properties showed that Av-Dex treatment maintained the bone volume at 3 weeks, while free Dex did not. Although trends towards reduced bone mineral density, trabecular number, thickness and increased spacing at 3 weeks were observed, with these parameters only becoming significant by week
9. Therefore, it was not possible to assess the effect of Dex or Av-Dex on these parameters. Abnormal joint loading (due to ACLT in this model) can lead to an adaptive bone remodelling process, decreasing trabecular bone volume fraction and thickness, due to an imbalance in bone resorption and formation in the more unloaded joint compartments (Florea et al., 2015). Finally, it was found that avidin penetrated through the complete thickness of articular cartilage and was retained within the tissue until at least 3 weeks (Fig. 11), thereby providing sustained release of Dex inside the cartilage. This was the most likely explanation for the greater reductions in measured inflammatory response and osteogenesis stimulation compared to free Dex. Both biomechanical and biochemical pathways could have a role to play in maintaining trabecular bone properties and inhibiting osteophyte manifestation in the Av-Dextreated group.

In the ACLT model of PTOA used in the current work, changes to the cartilage are first observed a
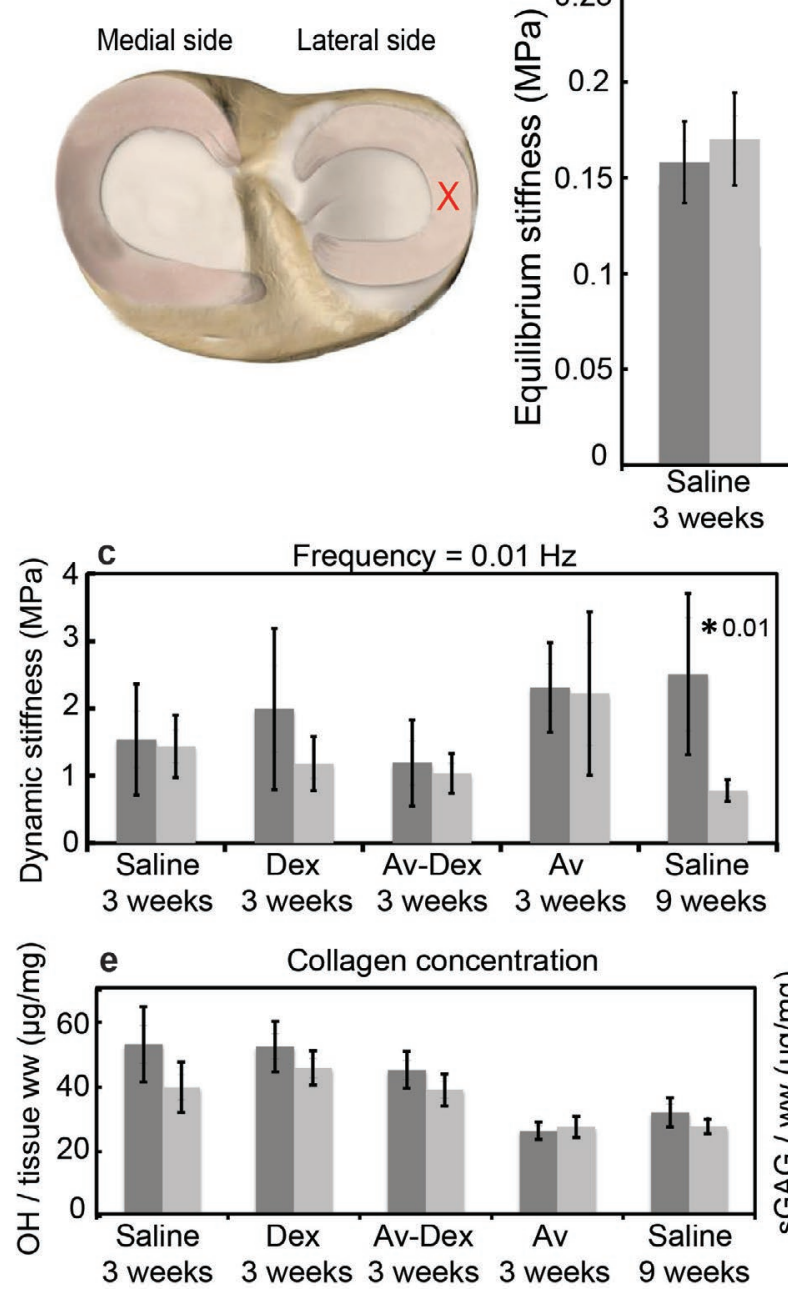

Contralateral control
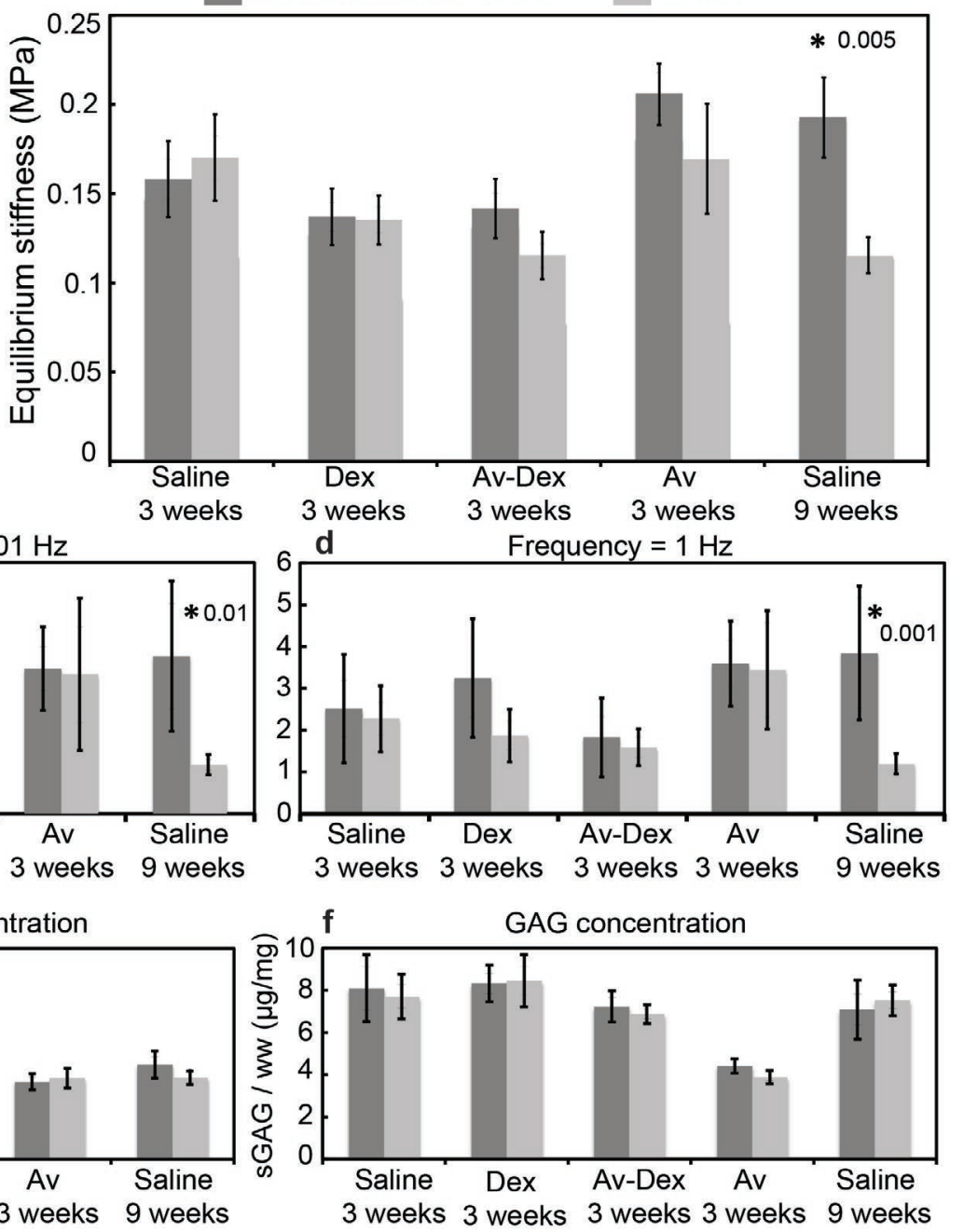

Fig. 8. Mechanical and biochemical properties of lateral meniscus. (a) Red cross represents the location tested on the lateral meniscus. (b) Average equilibrium stiffness values of lateral meniscus for each treatment group. Average dynamic stiffness measured in lateral meniscus at (c) $0.01 \mathrm{~Hz}$ and (d) $1 \mathrm{~Hz}$. (e) Collagen concentration and (f) GAG concentration measured in lateral meniscus. Data are shown as mean $\pm 95 \%$ CI; ${ }^{*}$ represents significant difference $(p<0.05)$ between ACLT and contralateral knee within each treatment group. $n=9-10$ joints in each group. 
within the medial femoral condyle and eventually extend to the lateral femoral condyle (Hayashi et al., 2008). Therefore, cartilage pooled from the medial side was used for gene expression analyses to elucidate early changes. Consistent with the literature, after ACLT transcriptional downregulation of ACAN and upregulation of MMP1, MMP3, MMP13 and ADAMTS5 (Bluteau et al., 2001; Chen et al., 2012; Hotta et al., 2005; Majima et al., 2002; Wu et al., 2008) were observed. Our findings agreed with the work of Hart and colleagues (Majima et al., 2002), who report a dramatic increase in MMP13 mRNA levels 3 weeks post ACLT in all cartilage regions, which then declined rapidly by 8 weeks, suggesting that MMP13 is involved in early remodelling of matrix in response to ACLT. Treatment with Dex and Av-
Dex reversed the downregulation of ACAN. AvDex significantly suppressed ILI $\beta$ and MMP1 gene expression, while free Dex did not. Suppression of pro-inflammatory IL-1 $\beta$ reduces COX2 and MMP production (Achari et al., 2012) that in turn can mediate the acute inflammatory response associated with surgically-induced osteoarthritis. Dex is shown to suppress MMP gene expression in IL1- $\alpha$-treated human cartilage explants (Li et al., 2015); however, in the current study, neither Dex nor Av-Dex suppressed $M M P 3$ or MMP13 expression. This may be due to the low Dex dose used. To our knowledge, there have not been any reports evaluating the effects of Dex on cartilage gene expression in a joint injury model.

While Av-Dex reduced the severity of arthritic changes in the trabecular bone and medial cartilage,

Contralateral control ACLT

a. Safranin O staining

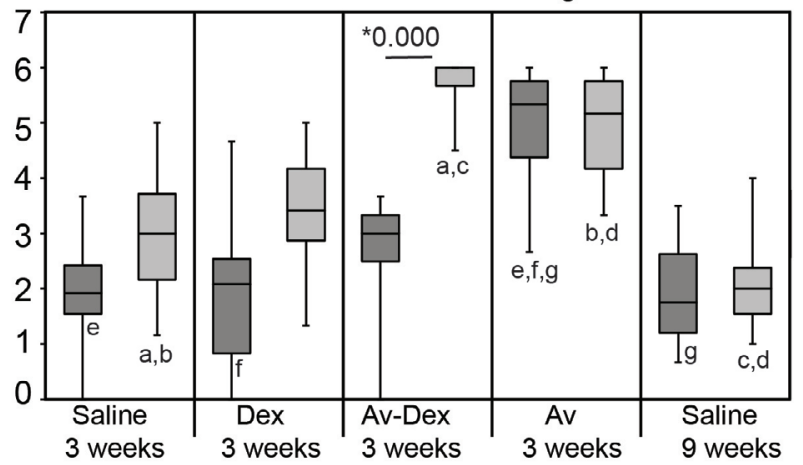

c. Chondrocyte density

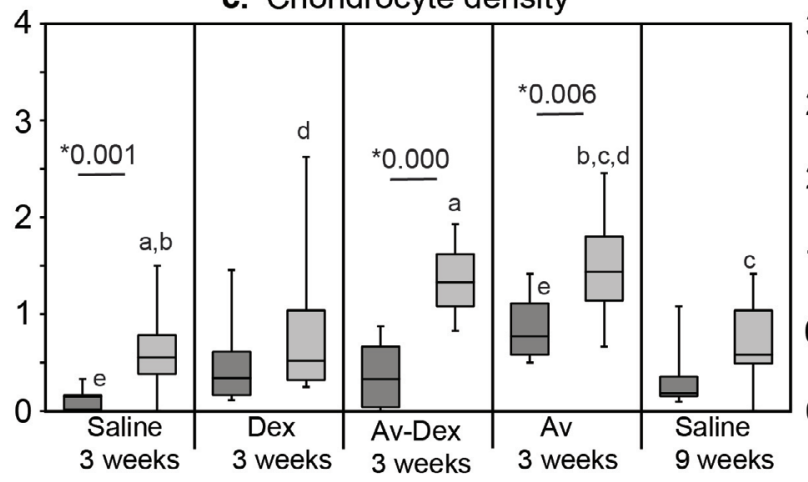

b. Surface structure

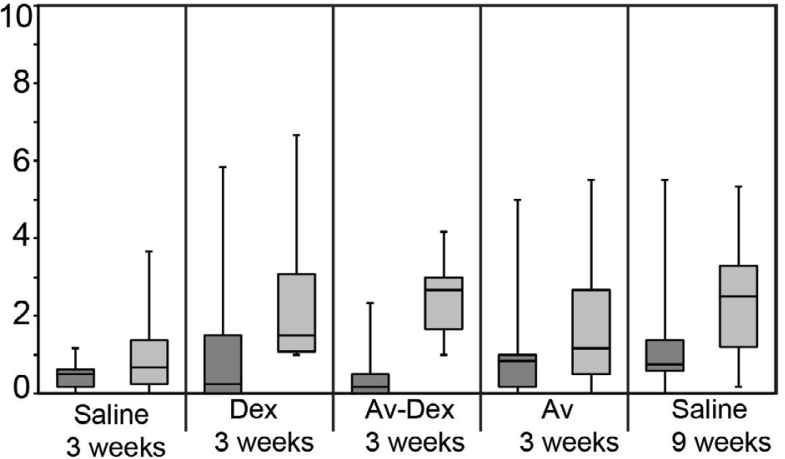

d. Chondrocyte clustering

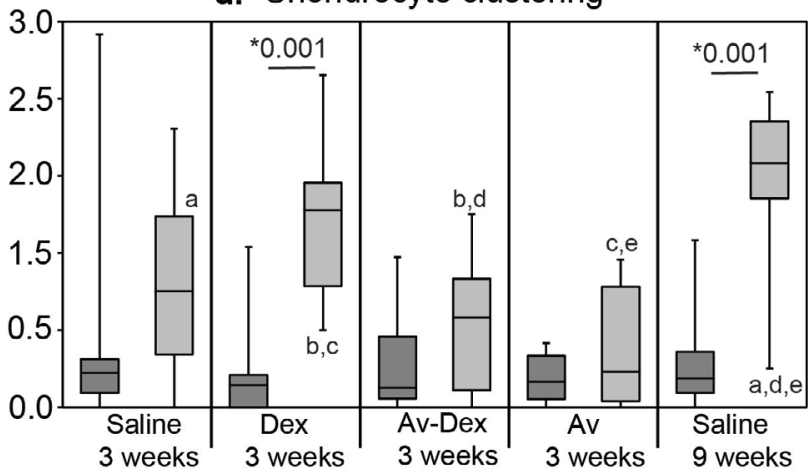

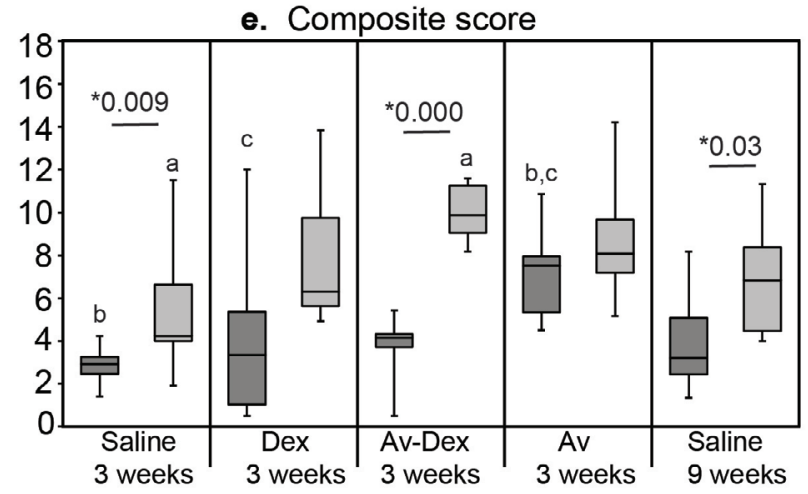

Fig. 9. Histological scoring of cartilage degeneration. Categorical scores as well as the composite histological score are presented as box-and-whisker plots ( $n=8-10$ condyles/group). Median values with $25^{\text {th }}-75^{\text {th }}$ quartile range are reported. * shows significant difference between ACLT and control within each treatment group using Mann Whitney U test. Groups that share letters within each graph are significantly different from one another ( $p<0.05$, Kruskal-Wallis ANOVA followed by post hoc Dunn's test). 

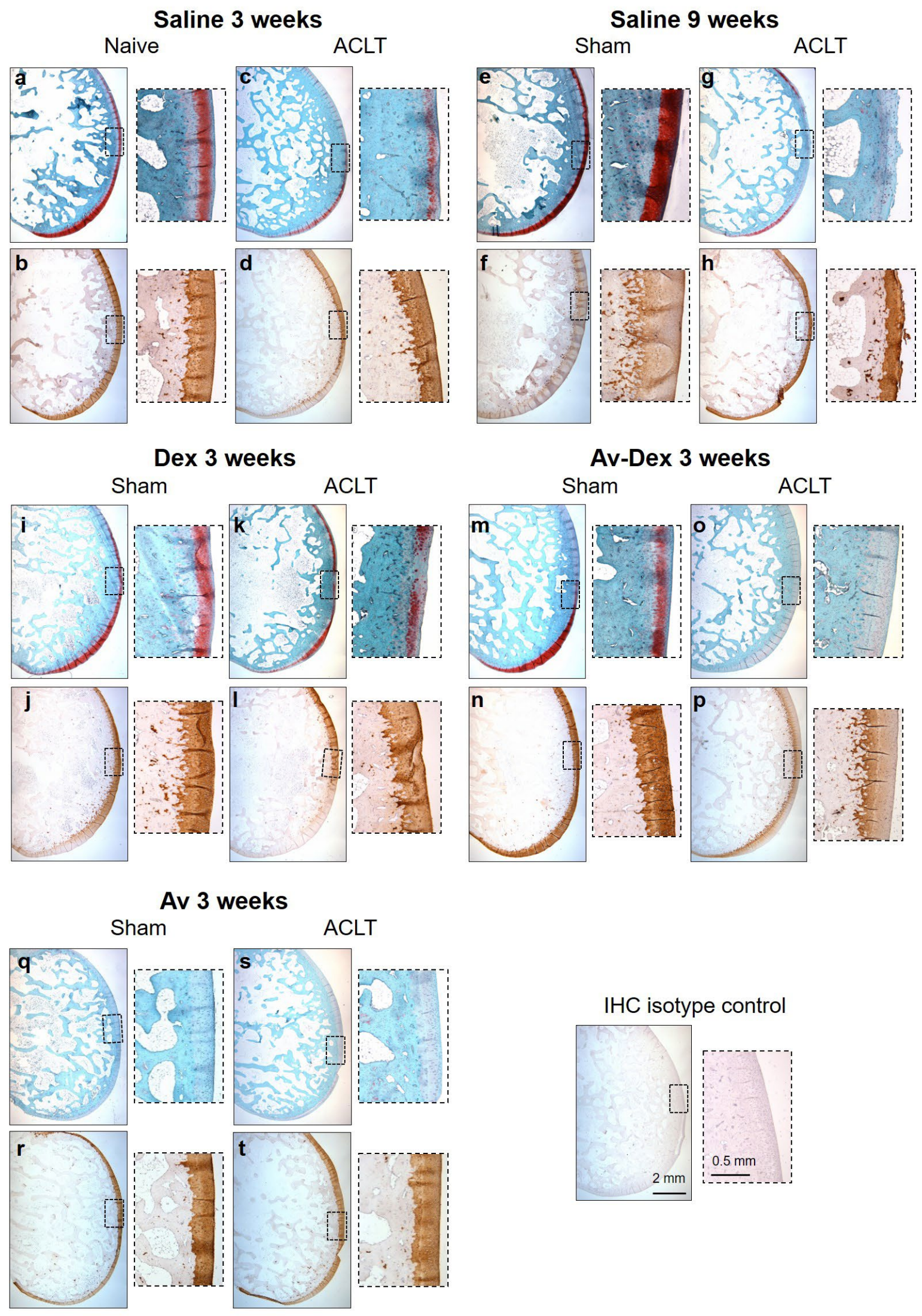

IHC isotype control

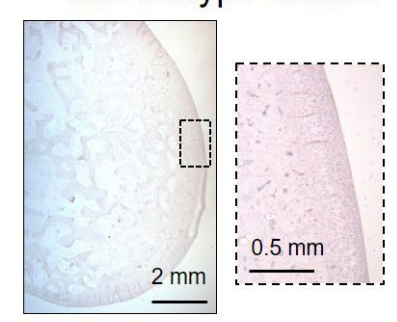

Fig. 10. Histology and collagen II IHC of sagittal sections taken from lateral condyles following ACLT or sham surgery. Serial sections were stained for safranin O/fast green (top rows) or immunostained for type II collagen (bottom rows). Insets show higher magnification images along the articular surface of the condyles. 


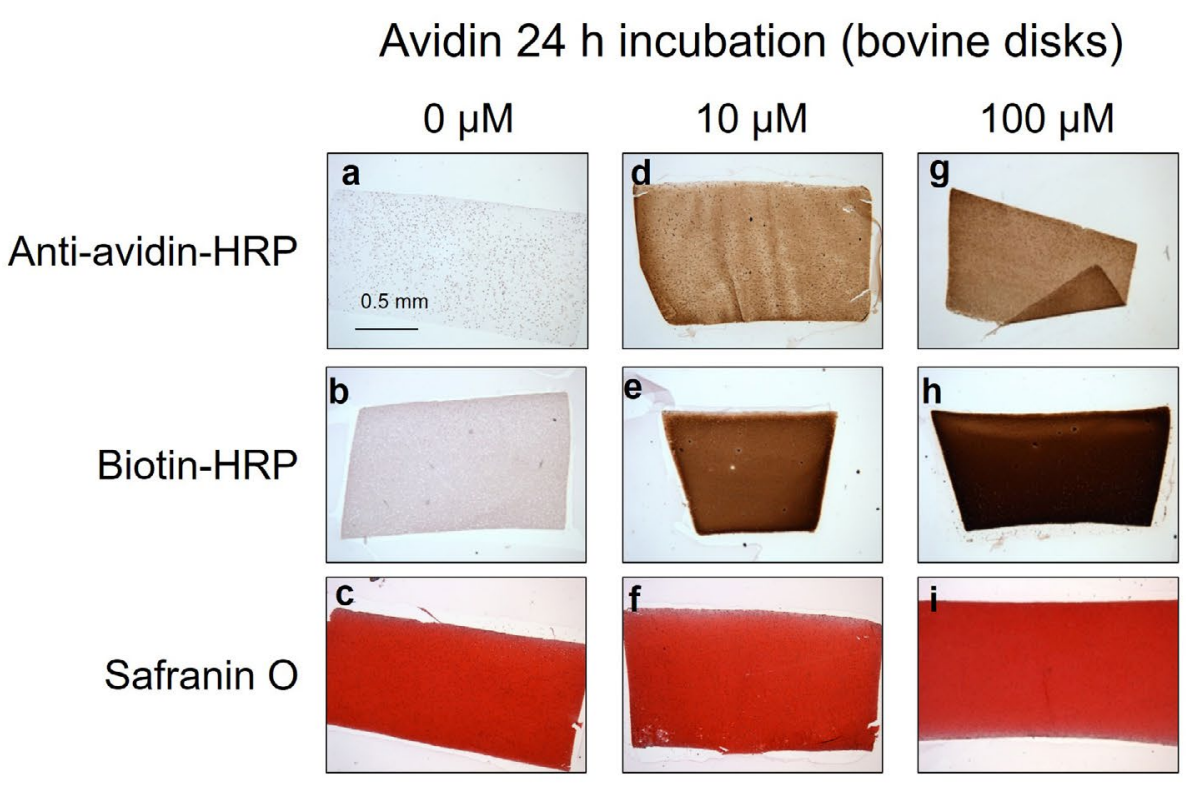

\section{Sham without $A v+D e x$}

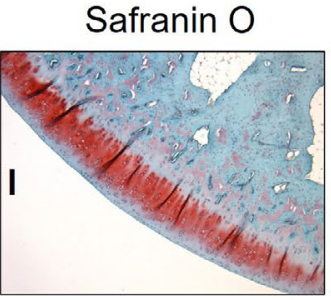

\section{Proximal} Condyle (near EDL enthesis)
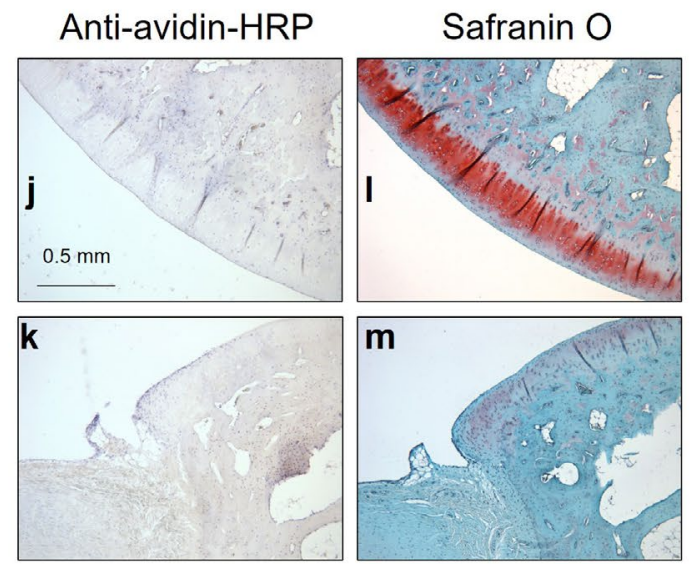

\section{Sham with avidin}

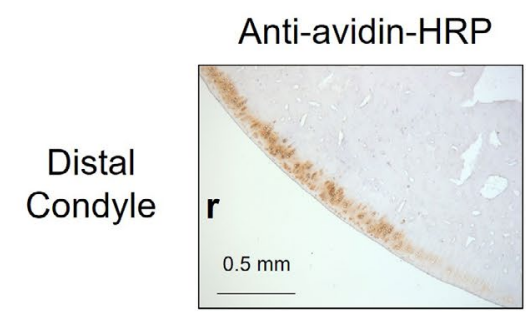

\section{Proximal Condyle (near EDL enthesis)}

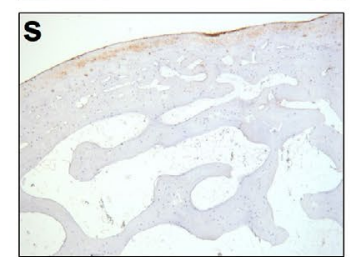

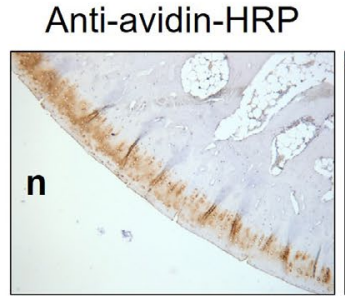
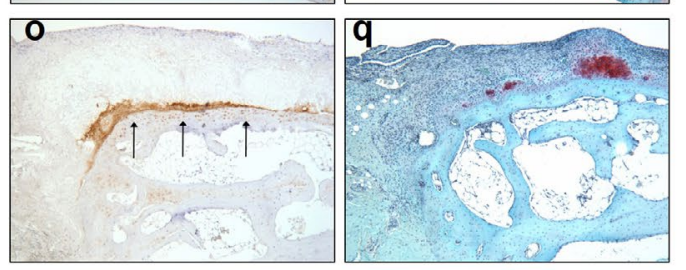

ACLT with avidin

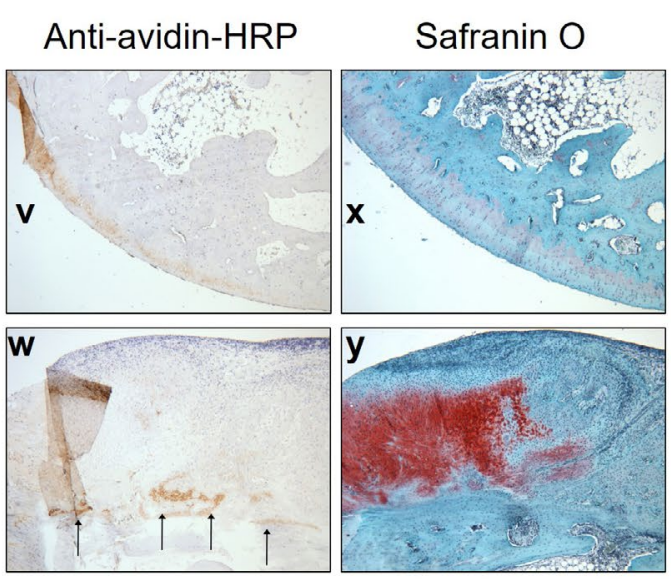

Fig. 11. Avidin immunostaining. (a-i) Specificity of the immunostaining protocol was validated using bovine cartilage disks, with or without $24 \mathrm{~h}$ pre-incubation in 10 or $100 \mu \mathrm{M}$ avidin prior to fixation. (j-y) Immunostaining of sagittal sections from rabbit lateral condyles. Specific signal was observed 2 weeks after injection within articular cartilage, which colocalised with safranin O staining. In the proximal condyle, near the enthesis of the EDL, immunostaining was detected near the location of the original articular surface (arrows), beneath layers of subsequent synovial infiltrate, chondrometaplasia and ossification. 
neither Av-Dex nor free Dex restored the ACLTinduced loss of mechanical stiffness and GAG content in lateral tibial cartilage at week 3. ACLT also resulted in GAG loss in the patellar cartilage that did not improve with Dex or Av-Dex treatment. Similar to articular cartilage, mechanical injuries can also alter the meniscus biomechanical properties, cause disorganisation of collagen network, tissue calcification and concomitant changes in GAG and collagen content (Katsuragawa et al., 2010; Kwok et al., 2014; Pauli et al., 2011; Sun et al., 2012). It was found that the changes in meniscus mechanical properties accompanied changes in the articular cartilage post ACLT; a significant loss (by almost a factor of two) of equilibrium and dynamic compressive stiffness in meniscus from the ACLT knee was observed at week 9, which is consistent with previous studies (Levillain et al., 2015). However, articular cartilage stiffness was significantly reduced at the earlier time point of 3 weeks post ACLT. Since no changes were observed in menisci at the 3-week time point, it was not possible to evaluate the effects of treatments. Loss in meniscus stiffness at 9 weeks might be attributed to a disordered collagen network or matrix calcification, as no changes in total GAG or collagen content were observed.

Av-Dex did not improve the composite histological score (Fig. 9e), primarily due to an increased safranin O sub-score that reflected the reduced GAG staining (Fig. 9a). This was consistent with the partial loss of GAG content ( 20-60\%) in lateral tibial and patellar cartilages (Fig. 7f,h), measured directly by DMMB assay. Also avidin alone caused a reduction in GAG staining within sham surgical controls (10-15 \% less compared to control saline 3 weeks, as measured by DMMB; Fig. 7f,h) and the effect was enhanced in the ACLT knees (Fig. 9a and Fig. 10i,j; 10-30 \% less GAG staining compared to saline 3 weeks ACLT as measured by DMMB, Fig. 7f,h). Together, the two assays suggested that GAG loss might be driven by the avidin dose $(20 \mathrm{mg})$ used in this study. Previously, it has been shown that $100 \mu \mathrm{M}$ avidin causes 2-fold larger GAG loss in bovine cartilage explants after $4 \mathrm{~d}$ incubation compared to control, although no GAG loss is observed for lower concentrations (Bajpayee et al., 2014a). The absence of safranin O staining does not necessarily indicate complete proteoglycan loss, given the known sensitivity issue reported for this histology dye (Camplejohn and Allard, 1988). This could explain the apparent discrepancy in GAG loss between safranin O staining and DMMB assay. It is noteworthy that some avidin remained in the cartilage at the 3-week endpoint (Fig. 11n,r,v). Consequently, it was not possible to rule out if this residual avidin contributed to reduce the safranin $\mathrm{O}$ staining by competing with the cationic dye for the binding to the negatively-charged GAG chains. Regarding long term cartilage health, it should be noted that the chosen avidin dose did not have any negative effects on cartilage surface structure, chondrocyte density or clustering compared to saline at 3 weeks (Fig. 9). Accordingly, it remains to be addressed whether any partial avidin-mediated GAG loss at 3 weeks was transient, such that GAG levels would return to previous levels as avidin would leave the cartilage.

A single injection of carrier-free Dex showed no improvement in early markers of joint degeneration, most likely due to its short residence time. It is well documented that the effects of glucocorticoids are dependent on the dose, frequency and duration of administration: large doses of corticosteroids have shown detrimental effects on cartilage structure, chondrocytes and subchondral bone (Wernecke et al., 2015). Only two reports were found that show chondroprotective effects with high Dex dose (Heard et al., 2015) and high frequency (Huebner et al., 2014) in a bone drill hole model of rabbit PTOA. A recent study by Flexion (Kumar et al., 2014) shows that intraarticular injection of clinically equivalent doses of triamcinolone in healthy beagle dogs $(2,6$ and $19 \mathrm{mg})$ causes significant GAG loss that started $3 \mathrm{~d}$ after IA injection and peaked at 4-6 weeks. However, this GAG loss started to recover by 6 months. Another recent human clinical trial shows that $40 \mathrm{mg}$ of triamcinolone administered IA every 3 months for 2 years results in significant loss of cartilage volume (McAlindon et al., 2017). Therefore, it is possible that the 3 weeks intra-cartilage signalling of Dex (despite its low dose), combined with a sufficiently high dose of avidin, could synergistically contribute to the partial GAG loss observed in this rabbit ACLT model.

Avidin alone resulted in a slight increase in the joint width of the contralateral knee, which was reflected in the $\mu \mathrm{CT}$ analysis of the osteophyte volume. However, it had no effect on the histological score evaluating synovial infiltration and morphogenesis (i.e. the extent of chondrometaplasia or endochondral ossification). This apparent discrepancy could be explained if synovial infiltration near the EDL enthesis was only associated with a certain threshold of osteophyte severity/volume.

Taken together, this study provided the first proof of concept towards the development of intra-cartilage delivery of small molecules, such as Dex, for treatment of PTOA. Although the study was performed with limited time points and with a single drug and carrier dosing, it successfully demonstrated that the cationic glycoprotein nanocarrier, avidin, could penetrate into cartilage and reside there for at least 3 weeks, thereby enabling sustained intra-cartilage delivery of Dex. Further studies are needed to optimise the dosing of drug as well as the carrier. The formulation used in the current study was comprised of $4 \mathrm{~mol}$ of Dex conjugated with $1 \mathrm{~mol}$ of avidin that, together, required about $20 \mathrm{mg}$ of avidin to deliver $0.5 \mathrm{mg}$ Dex. This potentially high dose of avidin could result in an intra-cartilage equilibrium uptake of $10-50 \mu \mathrm{M}$, depending on the tissue thickness (Bajpayee et al., 2015). At this concentration, avidin may begin to reduce intra-tissue osmotic pressure owing to its cationic charge, leading to decreased water content 
and potential loss of proteoglycans (Bajpayee et al., 2014a), explaining the partial loss of GAGs observed in avidin- or Av-Dex-treated groups. However, within bovine cartilage explants, avidin does not affect chondrocyte viability, biosynthesis of proteins and proteoglycans, even at such high doses (Bajpayee et al., 2014a). Ongoing work involves redesigning of the conjugate to increase its drug loading efficiency, such that a lower concentration of avidin is required to deliver the desired Dex dose.

The concentration-dependent effects of a drug on chondrocytes versus other intra-articular cell populations is of importance when selecting a dose for intra-cartilage delivery. While local drug concentrations will be highest near the chondrocytes using this delivery strategy (Bajpayee and Grodzinsky, 2017), the cartilage can also serve as a depot for drug release to the nearby synovium, meniscus and ligaments, albeit at lower concentrations. In the case of corticosteroids, which suppress catabolic activity in both chondrocytes and synoviocytes, the ideal drug-conjugate dose would release sufficient amounts of corticosteroid to inhibit pro-inflammatory signalling by synoviocytes, while limiting those cytotoxic effects on chondrocytes reported at higher concentrations (Dragoo et al., 2012). Anti-catabolic drugs targeting the synovium, but not cartilage, could also be used with this delivery approach, as long as chondrocytes are insensitive to concentrations higher than the effective synoviocyte dose. In contrast, this targeting strategy is ideally suited to use with cartilage anabolic drugs, such as growth factors: cartilage localisation would allow for these factors to be delivered at smaller doses that enhance chondrocyte ECM synthesis, while limiting adverse effects in the synovium, such as chondrometaplasia (Bakker et al., 2001; van Lent et al., 2004).

There were noteworthy limitations to the study design that potentially influenced the interpretation of the results. In order to minimise degradation of the ester and hydrazone linkers within the batch of Av-Dex conjugate, the experimental groups were executed in series, rather than randomising treatment assignment over the entire study cohort of 50 rabbits. Also, while all female rabbits were 12 months old, they were ordered in groups of 10 so that the surgical induction, injections and tissue harvests could be staggered. These choices potentially introduced variation among the treatment groups, due to subtle changes in subject history or model execution (e.g. surgical procedure, tissue harvest) with time. To help correct for such systemic variation, the contralateral knees were used as a reference when assessing most of the biological responses. However, for the avidin alone group, it was noted that the contralateral (sham) animals also received avidin, which limited the delineation of avidin-specific effects in this group, particularly on gene expression levels (Fig. 6). Another limitation concerns the focus on a 3-week endpoint for the test treatments, which prevented the assessment of how the test groups influenced longer-term joint changes. Because the biomechanical instability (due to ACLT) is not corrected in this model, it was decided to focus on early degenerative changes that could be prevented by a single, early injection of Dex: this approach modelled the critical clinical window between an ACL tear and arthroscopic repair. However, less degenerative changes were observed macroscopically in the lateral femoral condyle cartilage than in the medial femoral condyles at 3 weeks post-ACLT, in agreement with previous observations made in this rabbit model (Le Graverand et al., 2002; Saito et al., 2012). Because only lateral condyles were used for histology, this reduced the ability to ascertain treatment effects on cartilage degeneration by histological scoring. Despite these study limitations, protective effects against clinicallyrelevant markers of early joint degeneration were clearly demonstrated for avidin-mediated delivery of Dex.

Joint instability is the key factor in the onset and/ or progression of cartilage degradation in human PTOA and the ACLT model of PTOA used in this study mimicked that mechanism. Since this model alters both the magnitude and distribution of joint forces applied to the cartilage surface, it makes evaluation of the efficacy of structure-modifying OA drugs particularly challenging, because the instability that caused OA is still present even during therapeutic intervention (Hayashi et al., 2008). Future studies should consider drug intervention following mechanical stabilisation of the joint, which would permit evaluation of longer term effects of sustained delivery Dex and/or other promising DMOADs. For example, a combination therapy of Dex along with a pro-anabolic factor such as IGF-1 is shown to have a synergistic response in suppressing cartilage catabolism and stimulating proteoglycan biosynthesis in vitro (Grodzinsky et al., 2017; Li et al., 2015). An intra-cartilage delivery system using charge-charge (electrostatic) interactions, such as the one demonstrated in this study, can enhance drug penetration and transport into cartilage and enable sustained binding of drugs within the tissue's highly negatively charged matrix (Bajpayee and Grodzinsky, 2017). This can eventually enable clinical translation of DMOADs for treatment of early stage PTOA.

\section{Acknowledgements}

This work was funded in part by grants from the MIT Deshpande Centre for Technological Innovation, Klarman Family Foundation, NSF Materials Research Science and Engineering Centres (MRSEC) grant DMR1419807, NIH/NIBIB grant EB017755 and NIH/ NIAMS grants AR057105 and AR060331. We thank Dr David Hart and Dr Bryan Heard (University of Calgary) and Dr Tammy Haut-Donahue (Colorado State University) for their advice on elements of 
the rabbit model. Special thanks go to Dr Elisabeth Ferreira for guidance on qRT-PCR analysis.

\section{References}

Achari Y, Reno CR, Frank CB, Hart DA (2012) Carrageenan-induced transient inflammation in a rabbit knee model: molecular changes consistent with an early osteoarthritis phenotype. Inflamm Res 61: 907-914.

Anderson DD, Chubinskaya S, Guilak F, Martin JA, Oegema TR, Olson SA, Buckwalter JA (2011) Posttraumatic osteoarthritis: improved understanding and opportunities for early intervention. J Orthop Res 29: 802-809.

Bajpayee AG, Quadir MA, Hammond PT, Grodzinsky AJ (2016) Charge based intra-cartilage delivery of single dose dexamethasone using Avidin nano-carriers suppresses cytokine-induced catabolism long term. Osteoarthritis Cartilage 24: 71-81.

Bajpayee AG, Grodzinsky AJ (2017) Cartilagetargeting drug delivery: can electrostatic interactions help?. Nat Rev Rheumatol 13: 183-193.

Bajpayee AG, Scheu M, Grodzinsky AJ, Porter RM (2014a) Electrostatic interactions enable rapid penetration, enhanced uptake and retention of intraarticular injected avidin in rat knee joints. J Orthop Res 32: 1044-1051.

Bajpayee AG, Scheu M, Grodzinsky AJ, Porter RM (2015) A rabbit model demonstrates the influence of cartilage thickness on intra-articular drug delivery and retention within cartilage. J Orthop Res 33: 660667.

Bajpayee AG, Wong CR, Bawendi MG, Frank EH, Grodzinsky AJ (2014b) Avidin as a model for charge driven transport into cartilage and drug delivery for treating early stage post-traumatic osteoarthritis. Biomaterials 35: 538-549.

Bakker AC, van de Loo FA, van Beuningen HM, Sime P, van Lent PL, van der Kraan PM, Richards CD, van den Berg WB (2001) Overexpression of active TGF-beta-1 in the murine knee joint: evidence for synovial-layer-dependent chondro-osteophyte formation. Osteoarthritis Cartilage 9: 128-136.

Batiste DL, Kirkley A, Laverty S, Thain LMF, Spouge AR, Gati JS, Foster PJ, Holdsworth DW (2004) High-resolution MRI and micro-CT in an ex vivo rabbit anterior cruciate ligament transection model of osteoarthritis. Osteoarthritis Cartilage 12: 614-626.

Bluteau G, Conrozier T, Mathieu P, Vignon E, Herbage D, Mallein-Gerin F (2001) Matrix metalloproteinase-1, -3, -13 and aggrecanase-1 and -2 are differentially expressed in experimental osteoarthritis. Biochim Biophys Acta 1526: 147-158.

Bodick N, Lufkin J, Willwerth C, Kumar A, Bolognese J, Schoonmaker C, Ballal R, Hunter D, Clayman M (2015) An intra-articular, extendedrelease formulation of triamcinolone acetonide prolongs and amplifies analgesic effect in patients with osteoarthritis of the knee: a randomized clinical trial. J Bone Joint Surg Am 97: 877-888.

Bouchgua M, Alexander K, André d'Anjou M, Girard CA, Carmel EN, Beauchamp G, Richard H, Laverty S (2009a) Use of routine clinical multimodality imaging in a rabbit model of osteoarthritis - part I. Osteoarthritis Cartilage 17: 188-196.

Bouchgua M, Alexander K, Norman Carmel E, d'Anjou MA, Beauchamp G, Richard H, Laverty S (2009b) Use of routine clinical multimodality imaging in a rabbit model of osteoarthritis - part II: bone mineral density assessment. Osteoarthritis Cartilage 17: 197-204.

Boulocher C, Chereul E, Langlois JB, Armenean M, Duclos ME, Viguier E, Roger T, Vignon E (2007) Noninvasive in vivo quantification of the medial tibial cartilage thickness progression in an osteoarthritis rabbit model with quantitative 3D high resolution micro-MRI. Osteoarthritis Cartilage 15: 1378-1387.

Bouxsein ML, Boyd SK, Christiansen BA, Guldberg RE, Jepsen KJ, Müller R (2010) Guidelines for assessment of bone microstructure in rodents using micro-computed tomography. J Bone Miner Res 25: 1468-1486.

Brown TD, Johnston RC, Saltzman CL, Marsh JL, Buckwalter JA (2006) Posttraumatic osteoarthritis: a first estimate of incidence, prevalence, and burden of disease. J Orthop Trauma 20: 739-744.

Camplejohn KL, Allard SA (1988) Limitations of safranin "O" staining in proteoglycan-depleted cartilage demonstrated with monoclonal antibodies. Histochemistry 89: 185-188.

Chang DG, Iverson EP, Schinagl RM, Sonoda M, Amiel D, Coutts RD, Sah RL (1997) Quantitation and localization of cartilage degeneration following the induction of osteoarthritis in the rabbit knee. Osteoarthritis Cartilage 5: 357-372.

Chen W-P, Yu C, Hu P-F, Bao J-P, Tang J-L, Wu L-D (2012) Stigmasterol blocks cartilage degradation in rabbit model of osteoarthritis. Acta Biochim Pol 59: 537-541.

Chevalier X, Goupille P, Beaulieu AD, Burch FX, Bensen WG, Conrozier T, Loeuille D, Kivitz AJ, Silver D, Appleton BE (2009) Intraarticular injection of anakinra in osteoarthritis of the knee: a multicenter, randomized, double-blind, placebo-controlled study. Arthritis Care Res 61: 344-352.

Cohen S, Proudman S, Kivitz A, Burch F, Donohue J, Deborah B, Sun Y-N, Banfield C, Vincent M, Ni L, Zack D (2011) A randomized, double-blind study of AMG 108 (a fully human monoclonal antibody to IL-1R1) in patients with osteoarthritis of the knee. Arthritis Res Ther 13: R125.

Dong J, Jiang D, Wang Z, Wu G, Miao L, Huang L (2013) Intra-articular delivery of liposomal celecoxib-hyaluronate combination for the treatment of osteoarthritis in rabbit model. Int J Pharm 441: 285290.

Dragoo JL, Danial CM, Braun HJ, Pouliot MA, Kim HJ (2012) The chondrotoxicity of single-dose 
corticosteroids. Knee Surg Sports Traumatol Arthrosc 20: 1809-1814.

Evans CH (2016) Drug delivery to chondrocytes. Osteoarthritis Cartilage 24: 1-3.

Evans CH, Kraus VB, Setton LA (2014) Progress in intra-articular therapy. Nat Rev Rheumatol 10: 11-22.

Florea C, Malo MKH, Rautiainen J, Mäkelä JTA, Fick JM, Nieminen MT, Jurvelin JS, Davidescu A, Korhonen RK (2015) Alterations in subchondral bone plate, trabecular bone and articular cartilage properties of rabbit femoral condyles at 4 weeks after anterior cruciate ligament transection. Osteoarthritis Cartilage 23: 414-422.

Fortier LA, Balkman CE, Sandell LJ, Ratcliffe A, Nixon AJ (2001) Insulin-like growth factor-I gene expression patterns during spontaneous repair of acute articular cartilage injury. J Orthop Res 19: 720 728.

Gregory MH, Capito N, Kuroki K, Stoker AM, Cook JL, Sherman SL (2012) A review of translational animal models for knee osteoarthritis. Arthritis 2012: 764621.

Grodzinsky AJ, Wang Y, Kakar S, Vrahas MS, Evans CH (2017) Intra-articular dexamethasone to inhibit the development of post-traumatic osteoarthritis. J Orthop Res 35: 406-411.

Hashimoto S, Creighton-Achermann L, Takahashi K, Amiel D, Coutts RD, Lotz M (2002) Development and regulation of osteophyte formation during experimental osteoarthritis. Osteoarthritis Cartilage 10: $180-187$.

Hayashi M, Muneta T, Ju Y-J, Mochizuki T, Sekiya I (2008) Weekly intra-articular injections of bone morphogenetic protein-7 inhibits osteoarthritis progression. Arthritis Res Ther 10: R118.

Heard BJ, Barton KI, Chung M, Achari Y, Shrive NG, Frank CB, Hart DA (2015) Single intra-articular dexamethasone injection immediately post-surgery in a rabbit model mitigates early inflammatory responses and post-traumatic osteoarthritis-like alterations. J Orthop Res 33: 1826-1834.

Hoch DH, Grodzinsky AJ, Koob TJ, Albert ML, Eyre DR (1983) Early changes in material properties of rabbit articular cartilage after meniscectomy. J Orthop Res 1: 4-12.

Hotta H, Yamada H, Takaishi H, Abe T, Morioka H, Kikuchi T, Fujikawa K, Toyama Y (2005) Type II collagen synthesis in the articular cartilage of a rabbit model of osteoarthritis: expression of type II collagen C-propeptide and mRNA especially during early-stage osteoarthritis. J Orthop Sci 10: 595-607.

Huebner KD, Shrive NG, Frank CB (2014) Dexamethasone inhibits inflammation and cartilage damage in a new model of post-traumatic osteoarthritis. J Orthop Res 32: 566-572.

Hunziker EB, Kapfinger E, Geiss J (2007) The structural architecture of adult mammalian articular cartilage evolves by a synchronized process of tissue resorption and neoformation during postnatal development. Osteoarthritis Cartilage 15: 403-413.
Jones MD, Tran CW, Li G, Maksymowych WP, Zernicke RF, Doschak MR (2010) In vivo microfocal computed tomography and micro-magnetic resonance imaging evaluation of antiresorptive and antiinflammatory drugs as preventive treatments of osteoarthritis in the rat. Arthritis Rheum 62: 27262735.

Kapoor M, Martel-Pelletier J, Lajeunesse D, Pelletier J-P, Fahmi H (2011) Role of proinflammatory cytokines in the pathophysiology of osteoarthritis. Nat Rev Rheumatol 7: 33-42.

Katsuragawa Y, Saitoh K, Tanaka N, Wake M, Ikeda Y, Furukawa H, Tohma S, Sawabe M, Ishiyama M, Yagishita S, Suzuki R, Mitomi H, Fukui N (2010) Changes of human menisci in osteoarthritic knee joints. Osteoarthritis Cartilage 18: 1133-1143.

Kimmerling KA, Furman BD, Mangiapani DS, Moverman MA, Sinclair SM, Huebner JL, Chilkoti A, Kraus VB, Setton LA, Guilak F, Olson SA (2015) Sustained intra-articular delivery of IL-1RA from a thermally-responsive elastin-like polypeptide as a therapy for post-traumatic arthritis. Eur Cell Mater 29: 124-140.

Kumar A, Walz A, Garlick D, Spainhour C, Bodick N (2014) A single intra-articular dose of Fx006, an extended-release formulation of triamcinolone acetonide, has a similar effect on cartilage in healthy dogs as the commonly used kenalog- $40^{\circledR}$ suspension. Osteoarthritis Cartilage 22: S463-S464.

Kwok J, Grogan S, Meckes B, Arce F, Lal R, D'Lima D (2014) Atomic force microscopy reveals agedependent changes in nanomechanical properties of the extracellular matrix of native human menisci: implications for joint degeneration and osteoarthritis. Nanomedicine Nanotechnol Biol Med 10: 1777-1785.

Larsen C, Ostergaard J, Larsen SW, Jensen H, Jacobsen S, Lindegaard C, Andersen PH (2008) Intra-articular depot formulation principles: role in the management of postoperative pain and arthritic disorders. J Pharm Sci 97: 4622-4654.

Laverty S, Girard CA, Williams JM, Hunziker EB, Pritzker KPH (2010) The OARSI histopathology initiative - recommendations for histological assessments of osteoarthritis in the rabbit. Osteoarthritis Cartilage 18 Suppl 3: S53-65.

Le Graverand M-PH, Eggerer J, Vignon E, Otterness IG, Barclay L, Hart DA (2002) Assessment of specific mRNA levels in cartilage regions in a lapine model of osteoarthritis. J Orthop Res 20: 535544.

Levillain A, Boulocher C, Kaderli S, Viguier E, Hannouche D, Hoc T, Magoariec H (2015) Meniscal biomechanical alterations in an ACLT rabbit model of early osteoarthritis. Osteoarthritis Cartilage 23: 1186-1193.

Li Y, Wang Y, Chubinskaya S, Schoeberl B, Florine E, Kopesky P, Grodzinsky AJ (2015) Effects of insulinlike growth factor-1 and dexamethasone on cytokinechallenged cartilage: relevance to post-traumatic osteoarthritis. Osteoarthritis Cartilage 23: 266-274. 
Livak KJ, Schmittgen TD (2001) Analysis of relative gene expression data using real-time quantitative PCR and the 2(-Delta Delta C(T)) Method. Methods 25: 402-408.

Lu YC, Evans CH, Grodzinsky AJ (2011) Effects of short-term glucocorticoid treatment on changes in cartilage matrix degradation and chondrocyte gene expression induced by mechanical injury and inflammatory cytokines. Arthritis Res Ther 13: R142.

Majima T, Lo IKY, Randle JA, Marchuk LL, Shrive NG, Frank CB, Hart DA (2002) ACL transection influences mRNA levels for collagen type I and TNF- $\alpha$ in MCL scar. J Orthop Res 20: 520-525.

Malfait AM, Tortorella M, Thompson J, Hills R, Meyer DM, Jaffee BD, Chinn K, Ghoreishi-Haack N, Markosyan S, Arner EC (2009) Intra-articular injection of tumor necrosis factor-alpha in the rat: an acute and reversible in vivo model of cartilage proteoglycan degradation. Osteoarthritis Cartilage 17: 627-635.

Masoud I, Shapiro F, Kent R, Moses A (1986) A longitudinal study of the growth of the New Zealand white rabbit: Cumulative and biweekly incremental growth rates for body length, body weight, femoral length, and tibial length. J Orthop Res 4: 221-231.

McAlindon TE, LaValley MP, Harvey WF, Price LL, Driban JB, Zhang M, Ward RJ (2017) Effect of intra-articular triamcinolone $v s$. saline on knee cartilage volume and pain in patients with knee osteoarthritis: a randomized clinical trial. JAMA 317: 1967-1975.

Miller RE, Grodzinsky AJ, Cummings K, Plaas AHK, Cole AA, Lee RT, Patwari P (2010) Intraarticular injection of heparin-binding insulin-like growth factor 1 sustains delivery of insulin-like growth factor 1 to cartilage through binding to chondroitin sulfate. Arthritis Rheum 62: 3686-3694.

Pauli C, Grogan SP, Patil S, Otsuki S, Hasegawa A, Koziol J, Lotz MK, D’Lima DD (2011) Macroscopic and histopathologic analysis of human knee menisci in aging and osteoarthritis. Osteoarthritis Cartilage 19: 1132-1141.

Petit A, Redout EM, van de Lest CH, de Grauw JC, Müller B, Meyboom R, van Midwoud P, Vermonden T, Hennink WE, René van Weeren P (2015) Sustained intra-articular release of celecoxib from in situ forming gels made of acetyl-capped PCLA-PEGPCLA triblock copolymers in horses. Biomaterials 53: 426-436.

Pinney JR, Taylor C, Doan R, Burghardt AJ, Li X, Kim HT, Benjamin Ma C, Majumdar S (2012) Imaging longitudinal changes in articular cartilage and bone following doxycycline treatment in a rabbit anterior cruciate ligament transection model of osteoarthritis. Magn Reson Imaging 30: 271-282.

Saito M, Sasho T, Yamaguchi S, Ikegawa N, Akagi R, Muramatsu Y, Mukoyama S, Ochiai N, Nakamura J, Nakagawa K, Nakajima A, Takahashi K (2012) Angiogenic activity of subchondral bone during the progression of osteoarthritis in a rabbit anterior cruciate ligament transection model. Osteoarthritis Cartilage 20: 1574-1582.
Schmitz N, Laverty S, Kraus VB, Aigner T (2010) Basic methods in histopathology of joint tissues. Osteoarthritis Cartilage 18 Suppl 3: S113-116.

Shirai T, Kobayashi M, Nishitani K, Satake T, Kuroki H, Nakagawa Y, Nakamura T (2011) Chondroprotective effect of alendronate in a rabbit model of osteoarthritis. J Orthop Res 29: 1572-1577.

Sieker JT, Ayturk UM, Proffen BL, Weissenberger MH, Kiapour AM, Murray MM (2016) Immediate administration of intraarticular triamcinolone acetonide after joint injury modulates molecular outcomes associated with early synovitis. Arthritis Rheumatol 68: 1637-1647.

Sun Y, Mauerhan DR, Kneisl JS, James Norton $\mathrm{H}$, Zinchenko N, Ingram J, Hanley EN, Gruber HE (2012) Histological examination of collagen and proteoglycan changes in osteoarthritic menisci. Open Rheumatol J 6: 24-32.

Swärd P, Frobell R, Englund M, Roos H, Struglics A (2012) Cartilage and bone markers and inflammatory cytokines are increased in synovial fluid in the acute phase of knee injury (hemarthrosis) - a cross-sectional analysis. Osteoarthritis Cartilage 20: 1302-1308.

van der Kraan PM, van den Berg WB (2007) Osteophytes: relevance and biology. Osteoarthritis Cartilage 15: 237-244.

van Lent PLEM, Blom AB, van der Kraan $P$, Holthuysen AEM, Vitters E, van Rooijen N, Smeets RL, Nabbe KC a. M, van den Berg WB (2004) Crucial role of synovial lining macrophages in the promotion of transforming growth factor beta-mediated osteophyte formation. Arthritis Rheum 50: 103-111.

Wang SX, Laverty S, Dumitriu M, Plaas A, Grynpas MD (2007) The effects of glucosamine hydrochloride on subchondral bone changes in an animal model of osteoarthritis. Arthritis Rheum 56: 1537-1548.

Wernecke C, Braun HJ, Dragoo JL (2015) The effect of intra-articular corticosteroids on articular cartilage: a systematic review. Orthop J Sports Med 3: 2325967115581163.

Wu H, Du J, Zheng Q (2008) Expression of MMP-1 in cartilage and synovium of experimentally induced rabbit ACLT traumatic osteoarthritis: immunohistochemical study. Rheumatol Int 29: 3136.

Yoshioka M, Coutts RD, Amiel D, Hacker SA (1996) Characterization of a model of osteoarthritis in the rabbit knee. Osteoarthritis Cartilage 4: 87-98.

\section{Discussion with Reviewers}

John Kisiday: Is dexamethasone capable of suppressing all inflammatory pathways associated with PTOA?

Authors: Dex is a broad-spectrum glucocorticoid that interferes with cytokine-induced apoptotic signalling network by, for example, downregulating caspase-3 mRNA expression (Chae et al., 2000; Messmer et al., 1999; additional references). It is also known to 
inhibit cytokine-induced activation of the canonical NF-kB signalling pathway (Ray and Prefontaine, 1994; Scheinman et al., 1995; additional references), which transduces multiple pro-catabolic actions by inflammatory cytokines. However, no one factor, including dexamethasone, can inhibit the full spectrum of deleterious inflammatory pathways associated with the pathogenesis of OA. In the current study, Dex was used as an example of small molecule drug and, for future studies, it is worth to consider combining it with another (more specific) anti-inflammatory drug, such as IL-1RA.

John Kisiday: Is it possible to consider a different cationic carrier that does not negatively affect cartilage?

Authors: The high negative charge density of cartilage provides a unique opportunity to use charge interactions for enhancing the uptake, rate of penetration and retention of positively charged solutes. In this study, the premise that incorporating a positively charged carrier will improve intraarticular drug delivery was tested. Avidin was found to have an optimal size and charge to penetrate rapidly through full-thickness cartilage, resulting in 400 times higher uptake compared to its neutral counterpart. This high uptake also means that it is possible to lower the concentration of avidin for drug delivery, since most of it penetrated rapidly into the target tissue before clearing out from synovial fluid. However, in the current study, the dose of avidin was dictated by our conjugate design, where only 4 mol of Dex were conjugated to 1 mol of avidin using covalent linkers. The observed GAG loss was likely attributed to the high concentration of cationic avidin (20 mg) used to deliver $0.5 \mathrm{mg}$ of Dex. This side effect could potentially be eliminated by using alternative conjugation strategies that increase the molar ratio of Dex : avidin, a goal we are actively pursuing. It should be noted that highly positively charged solutes can change the Donnan osmotic swelling pressures of negatively charged tissues, causing loss of water molecules and GAGs; as consequence, this effect is likely not specific to avidin. Other materials, such as cationic dendrimers, peptides and polymeric particles, can be considered, but it is critical to use optimal charge range and concentration.

Robert Sah: Did the results suggest certain advantages or disadvantages of the avidin delivery system, compared to others that can attain sustained delivery? Authors: Advantages: avidin, due to its optimal size and charge, could rapidly penetrate through the full thickness of cartilage, bind within the tissue and provide sustained drug release close to the chondrocytes. No materials have yet been shown to penetrate through the dense cartilage matrix following intra-articular injection and deliver drugs inside the tissue. Most delivery techniques focus on increasing the residence time of drugs in the synovial fluid by using micron sized polymeric particles, aggregating hydrogels or peptides; however, these materials cannot penetrate the full depth of the tissue, which may be necessary, as most of cell and matrix target sites reside in the middle and deep zones of cartilage. Thus, avidin and other glycoproteins with similar size and charge provide a novel nanocarrier system for targeting cartilage and, likely, other negatively charged tissues.

Disadvantages: the results presented here suggested that it will be important to reduce the dose of avidin used for drug delivery. In the current study, $20 \mathrm{mg}$ of avidin were used to deliver $0.5 \mathrm{mg}$ of Dex, due to the low drug loading content $(2 \%$ by weight) achieved with the test conjugate. However, this delivery platform can be significantly improved by redesigning the conjugate to incorporate more sites on the protein or the PEG chains for covalent linking of drugs. This work is in progress.

\section{Additional references}

Chae HJ, Chae SW, Kang JS, Bang BG, Cho SB, Park RK, So HS, Kim YK, Kim HM, Kim HR (2000) Dexamethasone suppresses tumor necrosis factoralpha-induced apoptosis in osteoblasts: possible role for ceramide. Endocrinology 141: 2904-2913.

Messmer UK, Winkel G, Briner VA, Pfeilschifter J (2000) Glucocorticoids potently block tumour necrosis factor-alpha- and lipopolysaccharideinduced apoptotic cell death in bovine glomerular endothelial cells upstream of caspase 3 activation. Br J Pharmacol 127: 1633-1640.

Ray A, Prefontaine KE (1994) Physical association and functional antagonism between the p65 subunit of transcription factor NF-kappa B and the glucocorticoid receptor Proc Natl Acad Sci U S A 191: 752-756.

Scheinman RI, Gualberto A, Jewell CM, Cidlowski JA, Baldwin AS Jr (1995) Characterization of mechanisms involved in transrepression of NF-kappa $\mathrm{B}$ by activated glucocorticoid receptors. Mol Cell Biol 15: 943-953.

Editor note: The scientific editor for this paper was Martin Stoddart. 